\title{
A multiscale projection approach for the coupled global-local structural analysis of photovoltaic modules
}

\author{
M. Aßmus*, K. Naumenko, H. Altenbach \\ Chair of Engineering Mechanics, Institute of Mechanics, Faculty of Mechanical Engineering, Otto von Guericke University Magdeburg, \\ Universitätsplatz 2, 39106 Magdeburg, Germany
}

\begin{abstract}
The present contribution deals with the modeling and computational simulation of photovoltaic modules in the context of structural mechanics. Thereby, the focus is on the division of the boundary value problem of linear elastomechanics into two characteristic scales. The multiscale modeling starts at the global scale by means of an eXtended LayerWise Theory for a symmetric three-layered composite structure. A specially developed finite element is used to realise the discretisation. For the local structural analysis, a three dimensional unit cell is introduced which is representative in both plane directions and represents the structure of a photovoltaic module in transverse direction completely. The coupling of these two scales is carried out by the projection of the global deformations on the boundaries of the local structure, while the focus is on the transition from composite structure to three dimensional continuum. Thereby, characteristic coordinates for the location of a solar cell and an exemplary loading scenario are considered.

Overall, a modeling and simulation approach is presented which permits a numerically efficient solution of structuremechanical problems on photovoltaic modules through a sequential procedure of a deductive multiscale approach.
\end{abstract}

Keywords: photovoltaic module, multiscale, finite element analysis, layerwise theory, unit cell, projection

\section{Introduction}

\subsection{Motivation}

Structural mechanics analyses of photovoltaic modules gain increasing importance since the photovoltaic industry has a keen interest in the durability of their products, not least because electrical power losses can be correlated to mechanical failures, e.g. solar cell cracks and breakage [1,2]. Figure 1 indicates a typical photovoltaic module structure. Until now, high numerical expense of the predominantly 3D finite element analysis of whole photovoltaic modules in detail was needed. There, the majority of analyses is related to problems of elastostatics. Due to the extreme differences in material properties (e.g. the YounG's modulus $E^{c} \ll E^{s}, s$ stands for skin layers and $c$ for the core layer, respectively) of constituents and the relatively low thickness of the core layer compared to the skin layers $\left(h^{c} \ll h^{s}\right.$, where $h$ is the thickness of the corresponding layer), considerable effort on discretisation of the problem is required to gain sufficiently accurate results, resulting in a prohibitive amount of CPU time. Thus, the main drawback of this procedure is its high computational burden. Beside this, the problem of vanishing bending stiffness plays a decisive role which is not considered in standard solid finite elements definitions.

Due to the slenderness of a photovoltaic module $\left(H \ll L_{\min }\right.$, with the overall thickness $H=\sum h^{K}$ of the composite structure with $K$ layers and the minimum in-plane length $L_{\min }$ ), the mechanical contemplation allows a degeneration to a surface problem $[3,4,5]$ such that composite structure theories can be applied. Since the classical theories in this field, summarised e.g. in [6] and [7], like the first order shear deformation theory (FOSDT) fail to predict accurate results at the current problem [8,9], extensions of existing theories are required. Among many different formulations, zig-zag or layerwise theories [10, 11, 12] are mostly appropiate for the analysis of photovoltaic modules.

\footnotetext{
${ }^{*}$ Corresponding author

Email address: marcus . assmus@ovgu.de (M. Aßmus)
} 


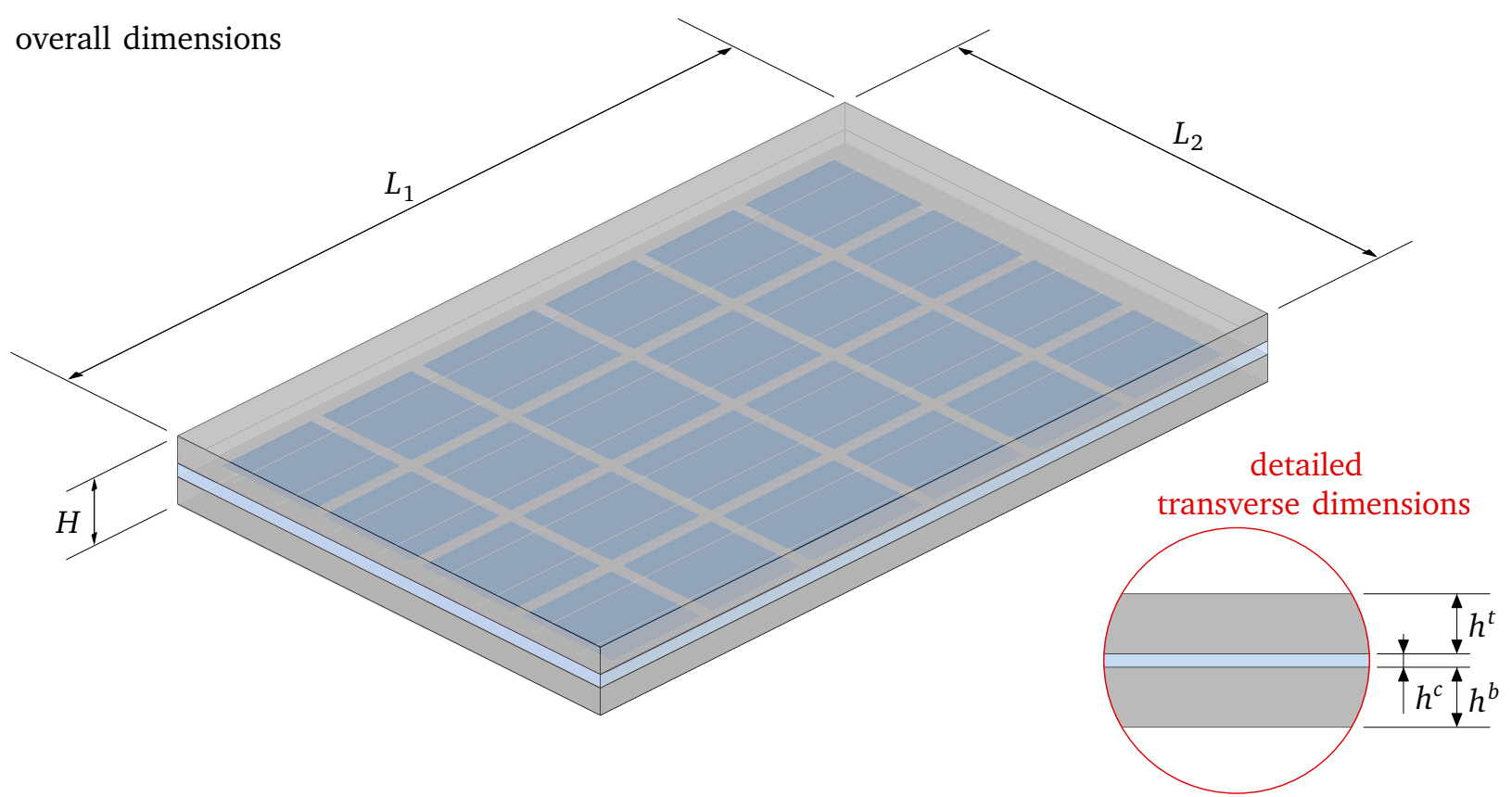

Figure 1: Longitudinal and transverse geometrical measures of the body $\mathfrak{B}$ mapped by a typical photovoltaic module structure (not to scale)

NAUMENKo \& EREMEYev [13] have developed an eXtended layerwise theory (XLWT) for such composite structures, called 'anti-sandwich' concerning the geometrical parameters in accordance to [8], which is also suitable for photovoltaic structures. Within this approach, balance and constitutive equations are derived for every layer individually. With constitutive assumptions for interaction forces and compatibility conditions, a model for the layered system is derived. Since a closed-form solution of this problem is concerned with restrictions to the boundary conditions which seem unrealistic for photovoltaic modules, a finite element has been introduced by EISENTRÄGER et al. [14] which allows the analysis to consider more realistic boundary conditions like framed edges with a significantly decreased computational effort compared to 3D FE models. Here, an enlargement based on a serendipity element has been developed under the assumptions of XLWT, which takes into account all layers, including all degrees of freedom. Through these two developments, an efficient procedure to solve the mechanical boundary value problem at global structure scale for realistic boundary conditions is introduced.

Nevertheless, there are also endeavors to analyse mechanical stresses at embedded solar cells, e.g. $[15,16]$, since the global structural analysis with the XLWT approach does not allow this. Hence, the present contribution carries out a novel approach using a scale separation by introducing a local structure and a projection strategy. For this purpose, a three dimensional unit cell, which is representative in the plane directions and completely determines a photovoltaic module in transverse direction, is used. By a deductive multiscale approach, the loading at global scale is transfered to local scale by introducing a multiscale projection method which permits a numerically efficient solution of the boundary value problem.

\subsection{Structure}

The aim of this paper is to present an efficient procedure for structural analyses of photovoltaic modules by partitioning the elastomechanic problem on two scales. For this purpose, a scale separation is introduced. At global scale, the structural analysis is focused on the deformation behaviour of the composite structure. At local scale, the analysis of mechanical stresses in the solar cells is in the foreground, where a unit cell is chosen due to the planar periodicity of the geometrical structure of the photovoltaic module. The whole procedure is visualised in Figure 2, while the paper is organised as follows:

In a first step, the structure, dimensions, and material properties of a typical photovoltaic module $\mathfrak{B}$ are presented (Figure 2, upper row far left). In the sequel, we limit our considerations to commercial crystalline silicon photovoltaic modules for terrestrial applications. Here, a symmetric structure in thickness direction with identical geometrical and 


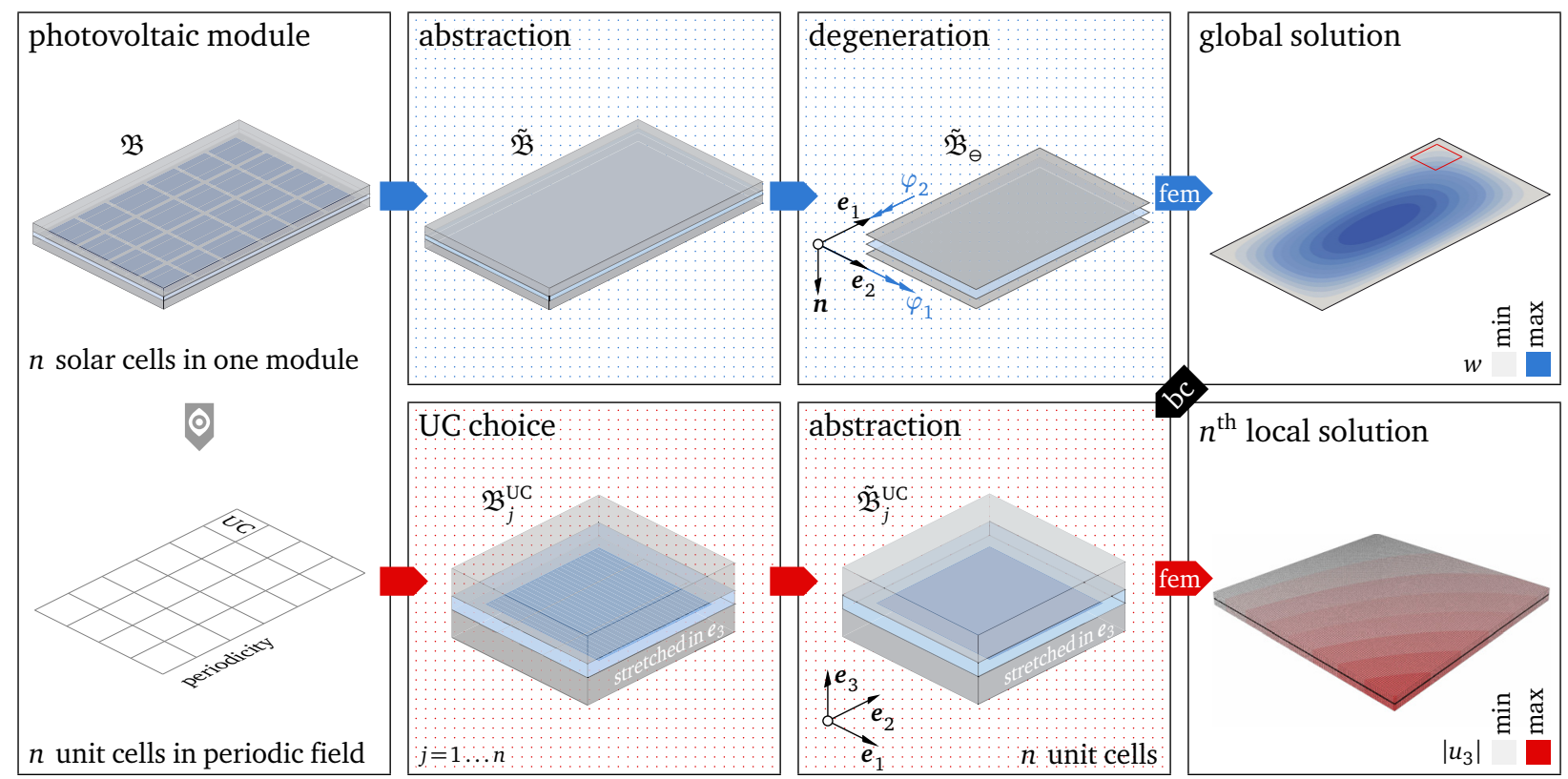

Figure 2: Structure of this work including all necessary steps to increase efficiency and accuracy (global: blue; local: red, scale transition: black)

physical parameters for the skin layers are used such that $h^{t}=h^{b}$ holds. A further limitation lies in the assumption of isotropic material behaviour for all layers such that the identities $E^{t}=E^{b}=E^{s}$ and $v^{t}=v^{b}=v^{s}$ can be specified, while $E^{c}$ and $v^{c}$ differ widely from $E^{s}$ and $v^{s}$. Based on this, an abstraction $\tilde{\mathfrak{B}}$ of the described photovoltaic module to a surface structure is carried out to obtain a mathematically solvable problem in the sense of thin-walled structure theories (Figure 2, upper row second graphic from left). For this purpose, the mathematical problem is degenerated to a midplane of every layer (Figure 2, upper row second graphic from right), where constraints to couple the layers are introduced. Here, a problem-oriented presentation of the underlying theory is presented. Attention is payed in particular to assumptions concerning boundary conditions. Here, the solution strategy with the Finite Element Method (FEM) is also scetched. As a result, a convergence study is conducted, and calculations of the deformation behaviour for different temperatures, which seem critical for the core layer material, are carried out (Figure 2, upper row far right). As consequence of the planar periodicity of a photovoltaic module (Figure 2, bottom row far left), a three dimensional unit cell $\mathfrak{B}^{\mathrm{UC}}$ (Figure 2, bottom row second from left) representing the local scale is defined, while the position of this unit cell in $\mathfrak{B}$ is arbitrarily selectable. This unit cell includes a whole solar cell in addition to the previously considered skin and the layers. To keep the problem as small as possible, simplifications have been introduced, too (Figure 2, bottom row second from right) such that an abstract unit cell $\tilde{\mathfrak{B}}^{\mathrm{UC}}$ is considered here. In order to prove the suitability of the unit cell for structural analysis, convergence tests based on three simple loading scenarios are presented.

As a logical step, a scale coupling has to be introduced to carry out realistic structural analyses of the stress state in a solar cell. Due to the information loss made at the abstraction and degeneration at global scale, assumptions for the way back from a composite structure to a 3D continuum are needed. Therefore, a projection method is introduced, where deformations of specific coordinates of a unit cell from the global solution are transferred to the local unit cell (Figure 2, 'bc' arrow). An approach for the transition of degrees of freedom is introduced since global and local scales are incompatible with respect to the degrees of freedom. After applying the projection for a sample position of the unit cell, a structural analysis is carried out for different temperatures resulting from global solution (Figure 2, bottom row far right). Here, the deformation behaviour of the unit cell and the resulting stresses on the embedded solar cell are discussed. Finally, indications of possible further work are given.

\subsection{Notation}

Throughout the whole work, a direct notation is preferred distinguishing between tensor and vector-matrix calculus. Zero rank tensors are symbolised by italic letters (e.g. $a$ ), first rank tensors by italic lowercase bold letters (e.g. $\boldsymbol{a}=a_{i} \boldsymbol{e}_{i}$ or $\boldsymbol{b}=b_{j} \boldsymbol{e}_{j}$ ), second rank tensors by italic uppercase bold letters (e.g. $\boldsymbol{A}=A_{l m} \boldsymbol{e}_{l} \otimes \boldsymbol{e}_{m}$ or $\boldsymbol{B}=B_{n o} \boldsymbol{e}_{n} \otimes \boldsymbol{e}_{o}$ ), and fourth rank 
tensors by italic uppercase bold calligraphic letters (e.g. $\mathcal{A}=A_{p q r s} \boldsymbol{e}_{p} \otimes \boldsymbol{e}_{q} \otimes \boldsymbol{e}_{r} \otimes \boldsymbol{e}_{s}$ ), where EINSTEIN sum convention is applied. Basic operations for tensors are the scalar product

$$
\boldsymbol{a} \cdot \boldsymbol{b}=a_{i} b_{j} \boldsymbol{e}_{i} \cdot \boldsymbol{e}_{j}=a_{i} b_{i}=\alpha \quad \alpha \in \mathbb{R},
$$

the cross product

$$
\boldsymbol{a} \times \boldsymbol{b}=a_{i} b_{j} \boldsymbol{e}_{i} \times \boldsymbol{e}_{j}=a_{i} b_{j} \epsilon_{i j k} \boldsymbol{e}_{k}=\boldsymbol{c},
$$

where $\epsilon_{i j k}$ is the LEVI-CIVITA-symbol

$$
\epsilon_{i j k}=\left\{\begin{aligned}
+1 & \text { if }(i, j, k) \text { is an even permutation of }(1,2,3) \\
-1 & \text { if }(i, j, k) \text { is an odd permutation of }(1,2,3) \\
0 & \text { if }(i, j, k) \text { is no permutation of }(1,2,3)
\end{aligned}\right.
$$

the dyadic product

$$
\boldsymbol{a} \otimes \boldsymbol{b}=a_{i} b_{j} \boldsymbol{e}_{i} \otimes \boldsymbol{e}_{j}=\boldsymbol{C},
$$

the composition of a second and a first rank tensor

$$
\boldsymbol{A} \cdot \boldsymbol{a}=A_{l m} a_{i} \boldsymbol{e}_{l} \otimes \boldsymbol{e}_{m} \cdot \boldsymbol{e}_{i}=A_{l i} a_{i} \boldsymbol{e}_{l}=\boldsymbol{d},
$$

the composition of two second rank tensors

$$
\boldsymbol{A} \cdot \boldsymbol{B}=A_{l m} B_{n o} \boldsymbol{e}_{l} \otimes \boldsymbol{e}_{m} \cdot \boldsymbol{e}_{n} \otimes \boldsymbol{e}_{o}=A_{l m} B_{m o} \boldsymbol{e}_{l} \otimes \boldsymbol{e}_{o}=\boldsymbol{D},
$$

the double scalar product between two second rank tensors

$$
\boldsymbol{A}: \boldsymbol{B}=A_{l m} B_{n o} \boldsymbol{e}_{l} \otimes \boldsymbol{e}_{m}: \boldsymbol{e}_{n} \otimes \boldsymbol{e}_{o}=A_{l m} B_{m l}=\beta \quad \beta \in \mathbb{R},
$$

and the double scalar product between a fourth and a second rank tensor

$$
\mathcal{A}: \boldsymbol{B}=A_{p q r s} B_{n o} \boldsymbol{e}_{p} \otimes \boldsymbol{e}_{q} \otimes \boldsymbol{e}_{r} \otimes \boldsymbol{e}_{s}: \boldsymbol{e}_{n} \otimes \boldsymbol{e}_{o}=A_{p q r s} B_{s r} \boldsymbol{e}_{p} \otimes \boldsymbol{e}_{q}=\boldsymbol{F}
$$

The transpose of the tensor $\boldsymbol{A}$ is defined as

$$
\boldsymbol{A}^{\top}=A_{m l} \boldsymbol{e}_{l} \otimes \boldsymbol{e}_{m}
$$

The vector invariant of a second rank tensor is introduced as

$$
\boldsymbol{A}_{\times}=\mathbf{1} \times \boldsymbol{A}=A_{l m} \boldsymbol{e}_{l} \times \boldsymbol{e}_{m} \quad \text { with } \mathbf{1}=\boldsymbol{e}_{i} \otimes \boldsymbol{e}_{i} .
$$

Using the tensor notation, latin indices (e.g. $i, j, k, l$ ) run through the values 1,2 , and 3 , while greek indices (e.g. $\alpha, \beta$ ) run through the values 1 and 2 . The vector valued Hamiltonian (Nabla operator) is defined as $\boldsymbol{\nabla}=\boldsymbol{e}_{\alpha}{ }^{\partial \ldots} / \partial X_{\alpha}$ for the surface considerations and $\boldsymbol{\nabla}=\boldsymbol{e}_{i}{ }^{\partial \ldots} / \partial x_{i}$ at three dimensional theory. $\boldsymbol{\nabla} \cdot \square$ is the divergence, and $\boldsymbol{\nabla} \square$ is the gradient of a tensor. $\nabla^{\mathrm{sym}} \square$ is the symmetric part of the associated gradient $\left({ }^{1} / 2\left[\nabla \square+\nabla^{\top} \square\right]\right)$, where $\square$ is a tensor of any rank. The transposed gradient is defined as $\nabla^{\top} \square=[\nabla \square]^{\top}$. For detailed penetrations of these operations, we refer to contemporary textbooks of continuum mechanics featuring mathematical propaedeutics for tensor calculus, e.g. Altenbach [17], Lai, Rubin \& Krempl [18], or Bertram [19]. In the present work, all considerations are restricted to CARTEsian coordinates with orthonormal bases $\boldsymbol{e}_{i}$ or $\boldsymbol{e}_{\alpha}$ and $\boldsymbol{n}$ respectively, in three dimensional Euclidean space $\mathbb{E}^{3}$. In what follows, we limit ourselves to geometrical and physical linearity at both scales.

In vector-matrix notation, vectors are denoted as upright lowercase sans serif bold letters (e.g. displacement vector $\mathbf{u}=\left[\begin{array}{lll}u_{1} & u_{2} & u_{3}\end{array}\right]^{\top}$ ) and matrices as upright uppercase sans serif bold letters (e.g. stiffness matrix $\mathbf{K}$ or matrix of shape functions $\mathbf{N}$ ). 


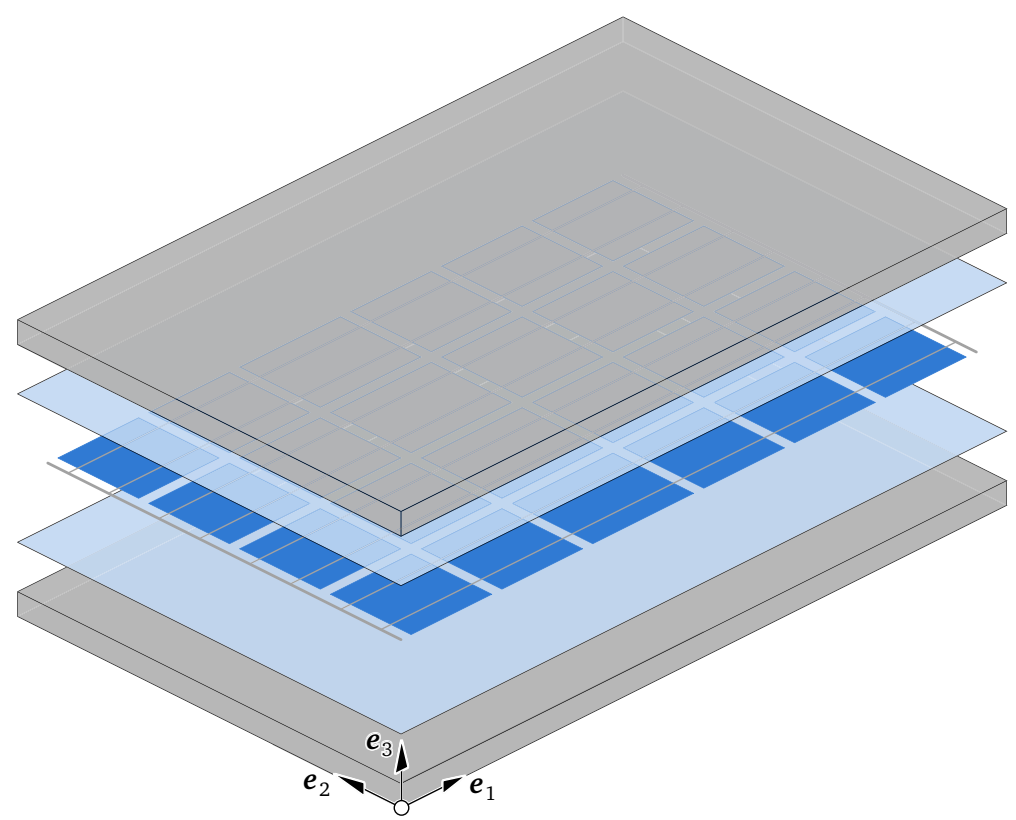

front/back cover encapsulant

solar cells

contacts

Figure 3: Mechanical layout of a crystalline silicon photovoltaic module $\mathfrak{B}$ with symmetric structure in transverse direction in exploded view

\section{Structure, dimensions, and material properties of photovoltaic modules}

A photovoltaic module is the main component of an energy conversion system that uses the semiconductor technology to convert light energy into electrical power in order to make it usable for power supply. The anatomy of a photovoltaic module restricted to symmetry in transverse direction is shown in Figure 3. The skin layers (front and back cover) are made of glass where their thicknesses are assumed to be equal in this work. In between there is a polymeric core layer, which in most cases consists of ethylene vinyl acetate (EVA). This encapsulant has to compensate different mechanical and thermal strains of the components and acts as buffer layer for the brittle silicon solar cells, which are embedded inside. For electrical wiring, various metallic contacts such as busbars and fingers are needed, which are, however, neglected in the context of the present treatise.

Due to lack of any geometrical dimensioning standardization in photovoltaic industry, a wide variety of dimensions is available on the market. Geometrical variations with a thickness ratio variation $T R=h^{c} /\left(h^{t}+h^{b}\right) \approx 0.125 \ldots 0.45$, a length ratio variation $L R=L_{2} / L_{1} \approx 0.25 \ldots 1$, and a thickness-to-length ratio variation $T L R=h / L_{\min } \approx 2 \cdot 10^{-3} \ldots 1.4 \cdot 10^{-2}$ are conceivable and partially available on the market. Considering common dimensions of a photovoltaic module, presented measures originate from a commercial photovoltaic module freely available, which will be concretised in the following.

Characteristic elastic material parameters for the main components are given in Table 1. Here, float glass for the skin layers is assumed. The copolymer EVA is the most widely used material as encapsulant in photovoltaic modules [20] and is therefore used here. The most common solar cells are made of polycrystalline silicon. For material parameters of polycrystalline silicon, the VoIGt-Reuss-Hill average [21, 22, 23] according to [24] is applied, using values for the monocrystals of crystalline silicon (cubic symmetry) taken from [25], where isotropic material behaviour results for macroscopic consideration due to the assumption that all crystals are of random orientation (respectiveley within a reasonable tolerance [26]) with a sufficiently large number of crystals in the investigated area [27].

Table 1: Elastic material parameters of the main components in a photovoltaic module at room temperature $\left(+23^{\circ} \mathrm{C}\right)$

\begin{tabular}{l|lll}
\hline material & float glass [28] & EVA [29] & polycrystalline silicon (based on [25]) \\
\hline$E\left[\mathrm{~N} / \mathrm{mm}^{2}\right]$ & $73.0 \cdot 10^{3}$ & 7.9 & $163.1 \cdot 10^{3}$ \\
$v[-]$ & 0.30 & 0.41 & 0.22 \\
\hline
\end{tabular}




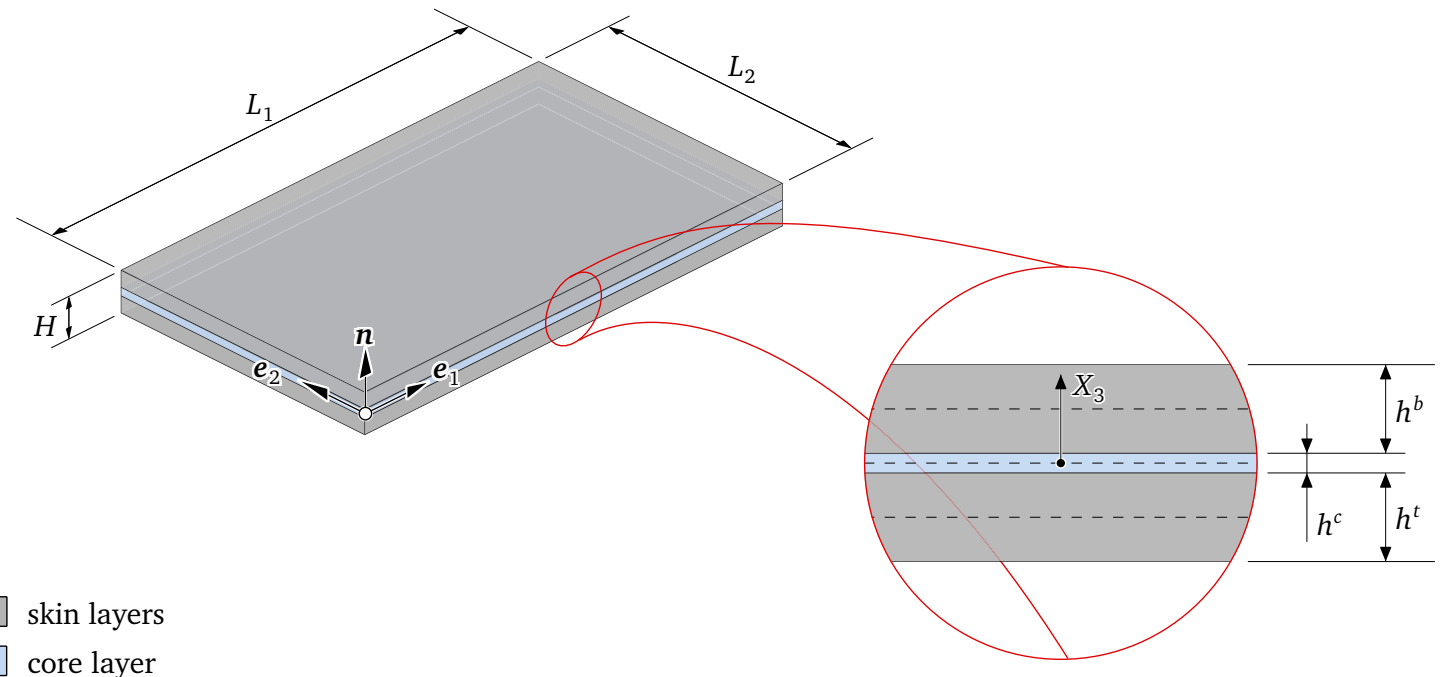

Figure 4: Design and dimensions of the abstract layerwise structure $\tilde{\mathfrak{B}}$ for global structural analysis with XLWT in accordance with [13]

\section{Global stuctural analysis}

\subsection{Assumptions and abstraction}

Naumenko \& ERemeyev [13] postulated that the solar cells in crystalline photovoltaic modules do not contribute essentially to the overall stiffness of the composite structure. Considering the core layer in transverse shear state, the solar cells do not constribute to the shear stiffness. When imagining the composite structure in tension or compression state, the stiffness is determined by the stiff skin layers such that the solar cells have only an insignificant influence on the membrane stiffness. Based on these reasons, the solar cells are neglected, and a homogeneous core layer is introduced whose mechanical behaviour is solely represented by the encapsulant material. The resulting abstract structure $\tilde{\mathfrak{B}}$ is visualised in Figure 4.

The treatment of the mechanical problem at global scale initially involves a reduction of the perspective from a three dimensional body to a surface continuum $\tilde{\mathfrak{B}}_{\ominus}$ in three dimensional space which has a thickness assigned individually. For this purpose, the individual layers visualised in Figure 4 can be reduced to a middle surface, indicated as dashed lines there. The origin of the individual layer coordinate systems $\left\{\boldsymbol{e}_{1}, \boldsymbol{e}_{2}, \boldsymbol{n}\right\}^{K}$ in transverse direction is located in the midplane of each layer, while the global coordinates origin is in the middle of the core layer such that $-{ }^{H} / 2 \leq X_{3} \leq{ }^{H} / 2$ (or $-h^{t}-h^{c} / 2 \leq X_{3} \leq h^{c} / 2+h^{b}$ with layer index $K=\{t, c, b\}$, see Figure 4) holds true. The description of material points $\boldsymbol{x}_{\ominus}^{K} \in \tilde{\mathfrak{B}}_{\ominus}$ at the composite layer midplanes is therefore given by the following expressions

$$
\boldsymbol{x}_{\ominus}^{K}=\boldsymbol{P} \cdot \boldsymbol{x}^{K}=X_{\alpha}^{K} \boldsymbol{e}_{\alpha} \quad \boldsymbol{x}^{K}=\boldsymbol{x}_{\ominus}^{K}+X_{3}^{K} \boldsymbol{n}
$$

where $\boldsymbol{x}_{\ominus}^{K}=\boldsymbol{P} \cdot \boldsymbol{x}^{K}$ is the planar projection of the three dimensional position vector, while $\boldsymbol{P}=\boldsymbol{e}_{\alpha} \otimes \boldsymbol{e}_{\alpha}=\mathbf{1}-\boldsymbol{n} \otimes \boldsymbol{n}$ is the metric tensor. Kinematical assumptions based on MindLIN theory with five degrees of freedom per layer $\left(\boldsymbol{u}_{\ominus}^{K}, w^{K}, \boldsymbol{\varphi}^{K} \Rightarrow\right.$ three translations, two rotations) are introduced, which is a pragmatic approach (see [30]) of the linear planar CosSERAT surface [31].

$$
\begin{aligned}
& \boldsymbol{u}^{K}=\boldsymbol{u}_{\ominus}^{K}+w^{K} \boldsymbol{n} \\
& \tilde{\boldsymbol{\varphi}}^{K}=\varphi_{\alpha}^{K} \boldsymbol{e}_{\alpha}+\varphi_{3}^{K} \boldsymbol{n} \\
& \left.\begin{array}{l}
\boldsymbol{u}_{\ominus}^{K}=\boldsymbol{P} \cdot \boldsymbol{u}^{K}=u_{\alpha}^{K} \boldsymbol{e}_{\alpha} \\
\boldsymbol{\varphi}^{K}=-\tilde{\boldsymbol{\varphi}}^{K} \times \boldsymbol{n}=-\varphi_{2}^{K} \boldsymbol{e}_{1}+\varphi_{1}^{K} \boldsymbol{e}_{2}
\end{array}\right\} \forall \boldsymbol{x}_{\ominus}^{K} \in \tilde{\mathfrak{B}}_{\ominus}
\end{aligned}
$$

Herein, $\boldsymbol{u}_{\ominus}^{K}=u_{\alpha}^{K} \boldsymbol{e}_{\alpha}$ are the in-plane displacements, $\varphi^{K}=-\varphi_{\alpha}^{K} \boldsymbol{e}_{\alpha} \times \boldsymbol{n}$ are the cross-section rotations and $w^{K}$ are the deflections. In summary, the system can now be described with five degrees of freedom per layer (two in-plane 
Table 2: Geometrical dimensions of the layerwise structure used to abstract a photovoltaic module

\begin{tabular}{l|lllll}
\hline parameter & $L_{1}[\mathrm{~mm}]$ & $L_{2}[\mathrm{~mm}]$ & $h^{t}[\mathrm{~mm}]$ & $h^{c}[\mathrm{~mm}]$ & $h^{b}[\mathrm{~mm}]$ \\
\hline value & 1620.0 & 810.0 & 3.2 & 1.0 & 3.2 \\
\hline
\end{tabular}

translational $u_{1}^{K}, u_{2}^{K}$, two rotational $\varphi_{1}^{K}, \varphi_{2}^{K}$, and one out-of-plane translational $w^{K}$ ). Geometrical dimensions used in the present study are summarised in Table 2 . The selected dimensions are therefore merely exemplary but however, based on the geometry of a real photovoltaic module with 72 solar cells.

\subsection{EXtended LayerWise Theory}

The XLWT allows us to analyse mechanical problems at the three-layered composite structure with thick, relatively stiff skin layers and a relatively thin and compliant core layer. Due to the simplifications presented in the previous section, it is also suitable for the photovoltaic module described in Chapter 2. Herinafter, the basic principles of this theory are presented in accordence with [13] where the governing equations based on a five-parametric linear theory of plates are defined layerwise. In contrast to the work of NAUMENKo \& EREMEYEv, a condensed representation reduced to static loading, orthogonal to the plate midlayer is shown, while linear elastic solutions of isotropic materials are in the foreground.

\subsubsection{Equilibrium conditions}

As basis for our purposes serves the first and second EuLER's law of motion [30, 32]. In case of elastostatic problems, the following equilibrium conditions in local form result [33].

$$
\begin{aligned}
& \text { balance of momentum: } \quad \boldsymbol{\nabla} \cdot \boldsymbol{F}^{K}+\boldsymbol{f}^{K}=\mathbf{o} \\
& \text { balance of moment of momentum: } \boldsymbol{\nabla} \cdot \boldsymbol{M}^{K}+\boldsymbol{F}_{\times}^{K}+\boldsymbol{m}^{K}=\mathbf{0} \\
& \} \forall \boldsymbol{x}_{\ominus}^{K} \in \tilde{\mathfrak{B}}_{\ominus}
\end{aligned}
$$

Herin, $\boldsymbol{F}^{K}$ is the force tensor, $\boldsymbol{F}_{\times}^{K}$ is the vectorial invariant of $\boldsymbol{F}^{K}, \boldsymbol{M}^{K}$ is the axial tensor of moments, $\boldsymbol{f}^{K}$ is the vector of interacting forces, and $\boldsymbol{m}^{K}$ is the vector of interacting moments. For a clear representation, the force tensor $\boldsymbol{F}^{K}$ can be decomposed additively into the membrane force tensor $N^{K}$ and the vector of transverse shear forces $\boldsymbol{q}_{Q}^{K}$.

$$
\boldsymbol{F}^{K}=\boldsymbol{N}^{K}+\boldsymbol{q}_{Q}^{K} \otimes \boldsymbol{n} \quad \text { with } \quad \boldsymbol{N}^{K}=\boldsymbol{F}^{K} \cdot \boldsymbol{P} \quad \wedge \quad \boldsymbol{q}_{Q}^{K}=\boldsymbol{F}^{K} \cdot \boldsymbol{n}
$$

The vectorial invariant of the force tensor is determined by $\boldsymbol{F}_{\times}^{K}=\boldsymbol{q}_{Q}^{K} \times \boldsymbol{n}$. Based on free-body diagrams for individual layers of the three-layered composite structure, given in Figure 5, it is possible to determine the stress resultants. The membrane force tensor $\boldsymbol{N}^{K}$, the axial tensor of moments $\boldsymbol{M}^{K}$, and the shear force vector $\boldsymbol{q}_{Q}^{K}$ can be obtained by through-the-thickness integration of the corresponding stresses $T^{K}$ of the $K$-th layer.

$$
\begin{array}{ll}
\boldsymbol{N}^{K}=N_{\alpha \beta}^{K} \boldsymbol{e}_{\alpha} \otimes \boldsymbol{e}_{\beta} & N_{\alpha \beta}^{K}=\int_{Z^{K^{-}}}^{Z^{K^{+}}} T_{\alpha \beta}^{K} \mathrm{~d} X_{3} \\
\boldsymbol{q}_{Q}^{K}=Q_{\alpha}^{K} \boldsymbol{e}_{\alpha} & Q_{\alpha}^{K}=\int_{Z^{K^{-}}}^{Z^{K^{+}}} T_{\alpha 3}^{K} \mathrm{~d} X_{3} \\
\boldsymbol{M}^{K}=-M_{\alpha \beta}^{K} \boldsymbol{e}_{\alpha} \otimes \boldsymbol{e}_{\beta} \times \boldsymbol{n} & M_{\alpha \beta}^{K}=\int_{Z^{K^{-}}}^{Z^{+}} X_{3} T_{\alpha \beta}^{K} \mathrm{~d} X_{3}
\end{array}
$$

Here, the components of the three dimensional CAUchY stress tensor $\boldsymbol{T}^{K}=T_{\alpha \beta}^{K} \boldsymbol{e}_{\alpha} \otimes \boldsymbol{e}_{\beta}+T_{\alpha 3}^{K} \boldsymbol{e}_{\alpha} \otimes \boldsymbol{n}+T_{3 \alpha}^{K} \boldsymbol{n} \otimes \boldsymbol{e}_{\alpha}+T_{33}^{K} \boldsymbol{n} \otimes \boldsymbol{n}$ are used. Integration limits can be specified as follows.

$$
Z^{K^{+}}=\left\{\begin{array}{ll}
+^{h^{c}} / 2+h^{b} & \text { for } K=t \\
+^{h^{c}} / 2 & \text { for } K=c \\
-h^{c} / 2 & \text { for } K=b
\end{array} \quad Z^{K^{-}}= \begin{cases}+h^{h^{c}} / 2 & \text { for } K=t \\
-h^{c} / 2 & \text { for } K=c \\
-h^{c} / 2-h^{b} & \text { for } K=b\end{cases}\right.
$$



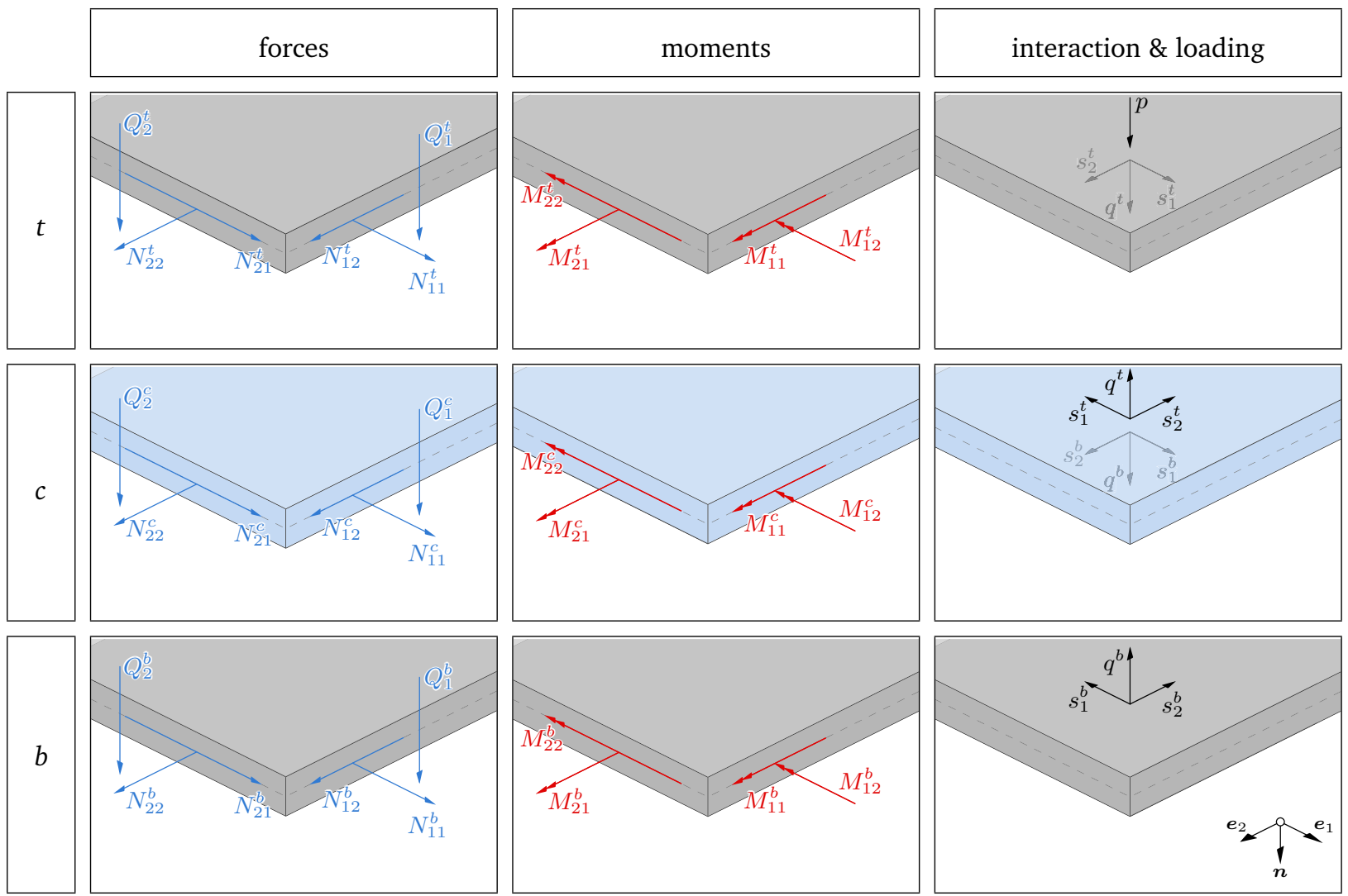

Figure 5: Stress resultants at midplane $\left(X_{3}^{K}=0\right)$ and interaction forces at front $\left(X_{3}^{K}=-h^{K} / 2\right)$ and back side $\left(X_{3}^{K}=+h^{K} / 2\right)$ of the individual layers

Concerning the interacting forces, the following relations can be deduced.

$$
\boldsymbol{f}^{K}=\left\{\begin{array}{cl}
\left(q^{t}+p\right) \boldsymbol{n}+\boldsymbol{s}^{t} & \text { for } K=t \\
\left(q^{b}-q^{t}\right) \boldsymbol{n}+\boldsymbol{s}^{b}-\boldsymbol{s}^{t} & \text { for } K=c \\
q^{b} \boldsymbol{n}-\boldsymbol{s}^{b} & \text { for } K=b
\end{array}\right.
$$

As well as decomposing the force tensor $\boldsymbol{F}^{K}$, it is also possible to split $\boldsymbol{f}^{K}$ additively into an in-plane $\boldsymbol{f}_{\ominus}^{K}$ and out-of-plane part $f_{\oplus}^{K}$.

$$
\boldsymbol{f}^{K}=\boldsymbol{f}^{K} \cdot(\boldsymbol{P}+\boldsymbol{n} \otimes \boldsymbol{n})=\boldsymbol{f}^{K} \cdot \boldsymbol{P}+\boldsymbol{f}^{K} \cdot \boldsymbol{n} \otimes \boldsymbol{n}=\boldsymbol{f}_{\ominus}^{K}+f_{\oplus}^{K} \boldsymbol{n}
$$

The interacting moments are determined by the following set of equations.

$$
\boldsymbol{m}^{K}= \begin{cases}\frac{h^{t}}{2} \boldsymbol{n} \times \boldsymbol{s}^{t} & \text { for } K=t \\ \frac{h^{c}}{2} \boldsymbol{n} \times\left(\boldsymbol{s}^{t}+\boldsymbol{s}^{b}\right) & \text { for } K=c \\ \frac{h^{b}}{2} \boldsymbol{n} \times \boldsymbol{s}^{b} & \text { for } K=b\end{cases}
$$

Variables used in Equations (19) and (21) are the in-plane interacting forces $\boldsymbol{s}^{K}=\boldsymbol{s}_{\alpha}^{K} \boldsymbol{e}_{\alpha}$ and the out-of-plane interacting forces $q^{K}$, while $p$ is the orthogonal loading at the outer surface of the top layer.Inserting Relations (14) to (21) in (13), we can determine the balances for the individual layer midplanes of the composite structure. The balance of membrane 
forces takes the following form

$$
\boldsymbol{\nabla} \cdot \boldsymbol{N}^{K}+\boldsymbol{f}_{\ominus}^{K}=\mathbf{0}
$$

The balance of transverse shear forces is indicated as follows.

$$
\boldsymbol{\nabla} \cdot \boldsymbol{q}_{Q}^{K}+f_{\oplus}^{K}=0 \quad f_{\oplus}^{K}=\left\{\begin{array}{cl}
q^{t}+p & \text { if } K=t \\
q^{b}-q^{t} & \text { if } K=c \\
-q^{b} & \text { if } K=b
\end{array}\right.
$$

Instead of the axial tensor of moments $\boldsymbol{M}^{K}$, the polar tensor of moments $\boldsymbol{L}^{K}=\boldsymbol{M}^{K} \times \boldsymbol{n}$ is introduced such that the balance of moments reads as follows.

$$
\boldsymbol{\nabla} \cdot \boldsymbol{L}^{K}-\boldsymbol{q}_{Q}^{K}+\boldsymbol{m}^{K} \times \boldsymbol{n}=\mathbf{0} \quad \boldsymbol{m}^{K} \times \boldsymbol{n}= \begin{cases}\frac{h^{t}}{2} \boldsymbol{s}^{t} & \text { if } K=t \\ \frac{h^{c}}{2}\left(\boldsymbol{s}^{t}+\boldsymbol{s}^{b}\right) & \text { if } K=c \\ \frac{h^{b}}{2} \boldsymbol{s}^{b} & \text { if } K=b\end{cases}
$$

\subsubsection{Constitutive and kinematical equations}

Considering a geometrically and physically linear theory, the constitutive equations for the stress resultants can be formulated layerwise as follows.

$$
\boldsymbol{N}^{K}=\mathcal{C}_{\ominus}^{K}: \boldsymbol{E}_{\ominus}^{K} \quad \boldsymbol{L}^{K}=\mathcal{D}^{K}: \boldsymbol{X}^{K} \quad \boldsymbol{q}_{Q}^{K}=\boldsymbol{Z}^{K} \cdot \boldsymbol{\gamma}^{K}
$$

Herein, $\boldsymbol{E}_{\ominus}^{K}$ is the membrane strain tensor, $\boldsymbol{X}^{K}$ is the curvature change tensor, and $\boldsymbol{\gamma}^{K}$ is the transverse shear strain vector. Under the assumption that strains and rotations remain small, these kinematical measures can be defined as follows.

$$
\boldsymbol{E}_{\ominus}^{K}=\nabla^{\mathrm{sym}} \boldsymbol{u}_{\ominus}^{K}=E_{\alpha \beta}^{K} \boldsymbol{e}_{\alpha} \otimes \boldsymbol{e}_{\beta} \quad \boldsymbol{X}^{K}=\boldsymbol{\nabla}^{\mathrm{sym}} \boldsymbol{\varphi}^{K}=\mathcal{X}_{\alpha \beta}^{K} \boldsymbol{e}_{\alpha} \otimes \boldsymbol{e}_{\beta} \quad \boldsymbol{\gamma}^{K}=\boldsymbol{\nabla} w^{K}+\boldsymbol{\varphi}^{K}=\gamma_{\alpha}^{K} \boldsymbol{e}_{\alpha}
$$

In Equation (26), the number of degrees of freedom for the three-layered composite structure under consideration is 15. The measures $\mathcal{C}_{\ominus}^{K}, \mathcal{D}^{K}$, and $\boldsymbol{Z}^{K}$ used in equation (25) are the fourth rank membrane stiffness tensor, the fourth rank bending stiffness tensor, and the second rank transverse shear stiffness tensor, respectively. In the case of isotropic material behaviour, these constitutive tensors read as follows [13, 14].

$$
\begin{aligned}
& \mathcal{C}_{\ominus}^{K}=D_{\mathrm{M}}^{K} v^{K} \boldsymbol{P} \otimes \boldsymbol{P}+D_{\mathrm{M}}^{K} \frac{1-v^{K}}{2}\left(\mathcal{P}_{1}+\mathcal{P}_{2}\right) \\
& \mathcal{D}^{K}=D_{\mathrm{B}}^{K} v^{K} \boldsymbol{P} \otimes \boldsymbol{P}+D_{\mathrm{B}}^{K} \frac{1-v^{K}}{2}\left(\mathcal{P}_{1}+\mathcal{P}_{2}\right) \\
& \boldsymbol{Z}^{K}=Z^{K} \boldsymbol{P}
\end{aligned}
$$

Here, $E^{K}, G^{K}$, and $v^{K}$ are the Young's modulus, the shear modulus, and the PoIsson's ratio of the corresponding layer material. The membrane stiffness $D_{\mathrm{M}}^{K}$, the bending stiffness $D_{\mathrm{B}}^{K}$ and the shear stiffness $Z^{K}$ can be determined as follows.

$$
D_{\mathrm{M}}^{K}=\frac{E^{K} h^{K}}{1-\left(v^{K}\right)^{2}} \quad D_{\mathrm{B}}^{K}=\frac{E^{K}\left(h^{K}\right)^{3}}{12\left[1-\left(v^{K}\right)^{2}\right]} \quad Z^{K}=\kappa^{K} G^{K} h^{K}
$$

$\mathcal{P}_{1}$ and $\mathcal{P}_{2}$ are tensors of fourth rank.

$$
\mathcal{P}_{1}=\boldsymbol{e}_{\alpha} \otimes \boldsymbol{e}_{\beta} \otimes \boldsymbol{e}_{\beta} \otimes \boldsymbol{e}_{\alpha} \quad \mathcal{P}_{2}=\boldsymbol{e}_{\alpha} \otimes \boldsymbol{e}_{\beta} \otimes \boldsymbol{e}_{\alpha} \otimes \boldsymbol{e}_{\beta}
$$

When determining the transverse shear stiffness in Equation (29), the shear correction factor $\kappa^{K}$ is needed. Basically $\kappa^{K}$ depends on the type of loading and boundary conditions. At present structure, the shear strains are transferred from the 
thick, shear rigid skin layers to the very thin core layer. Therefore, it is reasonable to assume that the shear stress in the thin core layer is nearly constant. As presented in [34], a value of $\kappa^{c}=1$ showed best agreements by comparing the theory presented here with a three dimensional FE solution. Experimental results presented in [35] affirm the postulated assumptions. Therefore, this value is used for the present calculations. Concerning the shear stiff skin layers, it holds $G^{t / b} \gg G^{c}$ and $h^{t / b} \gg h^{c}$ such that the choice of $\kappa^{K}$ is not decisive [36], therefore $\kappa^{K}=1 \forall K=\{t, b\}$ is also applied here.

\subsubsection{Kinematical constraints}

Assuming that we study a composite structure in virgin state where no delamination occurs and all layers are rigidly connected at any admissible deformation process, for in-plane displacements and cross-section rotations, the following kinematical constraints are deduced.

$$
\begin{gathered}
\boldsymbol{u}_{\ominus}^{t}+\frac{h^{t}}{2} \boldsymbol{\varphi}^{t}=\boldsymbol{u}_{\ominus}^{c}-\frac{h^{c}}{2} \boldsymbol{\varphi}^{c} \\
\boldsymbol{u}_{\ominus}^{b}-\frac{h^{b}}{2} \boldsymbol{\varphi}^{b}=\boldsymbol{u}_{\ominus}^{c}+\frac{h^{c}}{2} \boldsymbol{\varphi}^{c}
\end{gathered}
$$

In addition, since we neglect compression or dilatation in transverse direction, the equality of deflections of every layer is assumed.

$$
w^{t}=w^{c}=w^{b}=w \quad \forall \boldsymbol{x}_{\ominus}^{K}=X_{\alpha}^{K} \boldsymbol{e}_{\alpha}
$$

These assumptions are depicted in Figure 6 on the right hand side. For the sake of brevity, mean (superscript $\circ$ ) and relative (superscript $\Delta$ ) displacements and rotations are introduced.

$$
\begin{aligned}
\boldsymbol{u}^{\circ} & =\frac{1}{2}\left(\boldsymbol{u}_{\ominus}^{t}+\boldsymbol{u}_{\ominus}^{b}\right) & \boldsymbol{u}^{\Delta} & =\frac{1}{2}\left(\boldsymbol{u}_{\ominus}^{t}-\boldsymbol{u}_{\ominus}^{b}\right) \\
\boldsymbol{\varphi}^{\circ} & =\frac{1}{2}\left(\varphi^{t}+\varphi^{b}\right) & \boldsymbol{\varphi}^{\Delta} & =\frac{1}{2}\left(\varphi^{t}-\varphi^{b}\right)
\end{aligned}
$$

Considering the restrictions in Equations (32), (33), (34), (35), and (36), it is possible to reduce the number of overall degrees of freedom for the composite structure to nine: $u_{1}^{\circ}, u_{2}^{\circ}, u_{1}^{\Delta}, u_{2}^{\Delta}, w, \varphi_{1}^{\circ}, \varphi_{2}^{\circ}, \varphi_{1}^{\Delta}$, and $\varphi_{2}^{\Delta}$.

\subsection{Boundary Conditions}

The solution of the boundary value problem requires the determination of adequate boundaries where loads are applied and the structure is supported. For this purpose, the domain boundary is divided into support edges, where DIRICHLET boundary conditions and loaded areas, where Neumann boundary conditions are prescribed. Mixed boundary conditions are not considered here. The support edges are denoted as

$$
\Gamma_{\mathrm{De}} \forall \boldsymbol{x}_{0} \in\left\{\Gamma_{1}^{1}, \Gamma_{1}^{2}, \Gamma_{2}^{1}, \Gamma_{2}^{2}\right\} \quad\left\{\Gamma_{1}^{1}, \Gamma_{1}^{2}, \Gamma_{2}^{1}, \Gamma_{2}^{2}\right\} \in \Gamma_{\mathrm{De}} \subset \partial \tilde{\mathfrak{B}}_{\ominus},
$$

and the loaded area is denoted as $\Gamma_{\mathrm{Ne}}$, which is the outer face normal to the midplane of the front cover. Precise data indicating the edges can be found in Table 3.

Table 3: Definition of boundaries at the layerwise structure

\begin{tabular}{l|lllll}
\hline boundary & $\Gamma_{1}^{1}$ & $\Gamma_{1}^{2}$ & $\Gamma_{2}^{1}$ & $\Gamma_{2}^{2}$ & $\Gamma_{\mathrm{N}}$ \\
\hline $\boldsymbol{e}_{1}$ & 0 & $L_{1}$ & $0 \ldots L_{1}$ & $0 \ldots L_{1}$ & $0 \ldots L_{1}$ \\
$\boldsymbol{e}_{2}$ & $0 \ldots L_{2}$ & $0 \ldots L_{2}$ & 0 & $L_{2}$ & $0 \ldots L_{2}$ \\
$\boldsymbol{n}$ & $-{ }^{H} / 2 \ldots{ }^{H} / 2$ & $-{ }^{H} / 2 \ldots{ }^{H} / 2$ & $-{ }^{H} / 2 \ldots{ }^{H} / 2$ & $-{ }^{H} / 2 \ldots{ }^{H} / 2$ & $-{ }^{H} / 2$ \\
\hline
\end{tabular}


assembly in reference placement

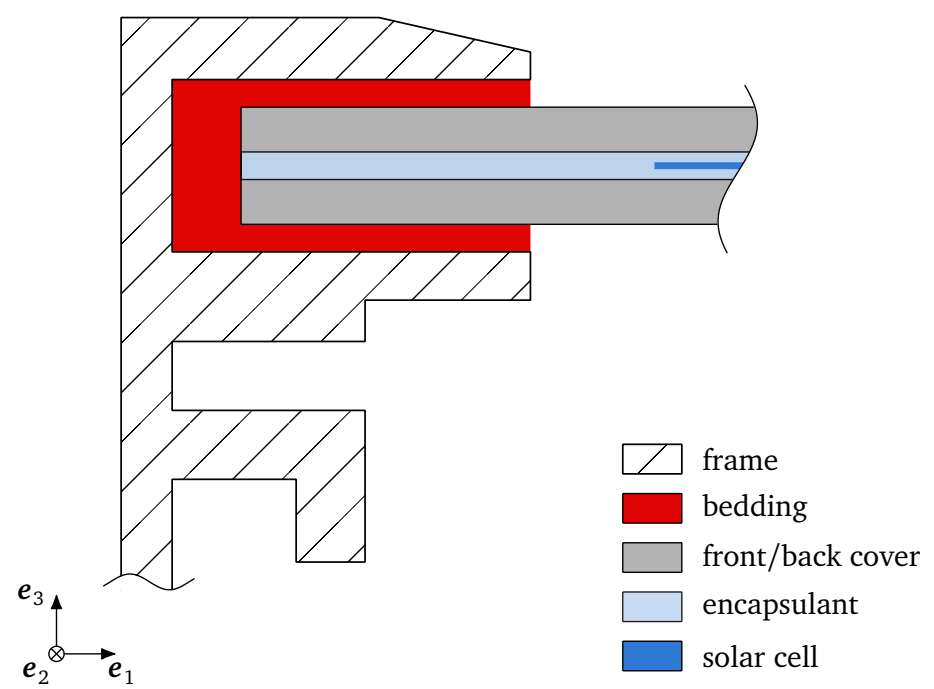

mechanical analogon in current placement

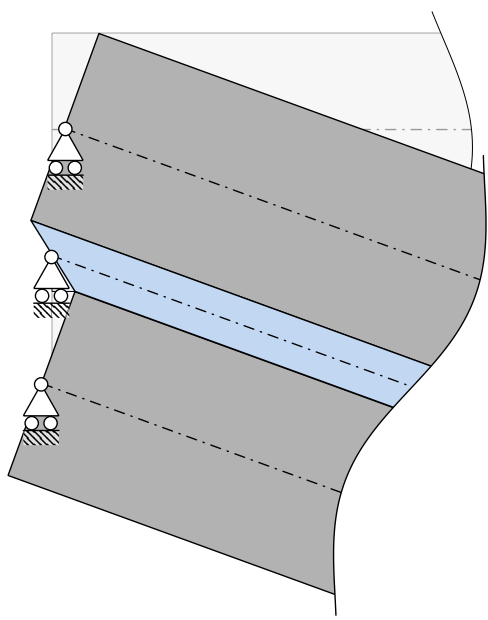

Figure 6: Mounting of photovoltaic composite in a frame and kinematical assumptions in deformed configuration (kinematics magnified)

\subsubsection{Edge support}

The support of photovoltaic composite structures is realised by aluminum profiles mounted peripherally in most cases. Here, the composite structure is inserted into a groove of the profile, which is provided with a soft bedding material. This is in most cases made of injected silicone or sealant tape. A detailed view of a typical support situation is given in Figure 6 (left side). In this work it is assumed that the layers of the composite structure can twist individually at the support edges and can move freely in plane directions. Due to the lack of information for the bedding material, the elastic foundation (e.g. WinKLER [37] or PASTERNAK [38] bedding)at this support is not included. Only deflections $w$ should therefore be disabled.

$$
w=0 \quad \forall \boldsymbol{x}_{\ominus} \in \Gamma_{\mathrm{De}}
$$

\subsubsection{Surface loading}

For the sake of simplicity, only orthogonal surface loads are considered here. The standard IEC 61215 [39] gives a first indication for the loading condition at photovoltaic modules. There, a homogeneous distributed load orthogonal to midplane is applied on the front cover surface, where the magnitude is $\pm 2.4 \cdot 10^{-3}$ or $\pm 5.4 \cdot 10^{-3} \mathrm{~N} / \mathrm{mm}^{2}$. For the sake of the restricted validity for the geometrically linear theory $\left({ }^{w_{\max }} / H \leq 0.5\right.$ ), a load of $p=0.5 \cdot 10^{-3} \mathrm{~N} / \mathrm{mm}^{2}$ in $\boldsymbol{n}$ direction is applied on the surface of the front cover (see Figure 4).

$$
p=p n \quad \forall x_{\ominus} \in \Gamma_{\mathrm{Ne}}
$$

\subsection{Discretisation and convergence of XLWT composite structure}

In order to solve the mechanical boundary value problem numerically, the spatial discretisation of the weak form by using the finite element method is necessary. The whole two dimensional domain $\Omega_{\mathrm{XLWT}}\left(\Omega_{\mathrm{XLWT}} \approx \tilde{\mathfrak{B}}_{\ominus}\right)$ is thus divided into several subdomains $\Omega_{\mathrm{XLWT}}^{e}$ where $e$ stands for element.

$$
\Omega_{\mathrm{XLWT}}=\bigcup_{e=1}^{N E} \Omega_{\mathrm{XLWT}}^{e}
$$$$
\Omega_{\mathrm{XLWT}}^{i} \cap \Omega_{\mathrm{XLWT}}^{j}=\emptyset \text { for } i \neq j
$$ 
where $N E$ is the number of elements. The virtual work of each element is calculated under the assumption that the sum of the virtual works in each subdomain equals the virtual work of the whole domain.

$$
\delta W_{\mathrm{int}}=\sum_{e=1}^{N E} \delta W_{\mathrm{int}}^{e} \quad \delta W_{\mathrm{ext}}=\sum_{e=1}^{N E} \delta W_{\mathrm{ext}}^{e}
$$

The vector of degrees of freedom per node $\mathbf{a}^{N}$ resulting from Section 3.2.3 is now written as follows.

$$
\mathbf{a}^{N}=\left[\begin{array}{lllllllll}
u_{1}^{\circ} & u_{2}^{\circ} & u_{1}^{\Delta} & u_{2}^{\Delta} & w & \theta_{1}^{\circ} & \theta_{2}^{\circ} & \theta_{1}^{\Delta} & \theta_{2}^{\Delta}
\end{array}\right]^{\top} \quad \theta_{1}^{R}=-\varphi_{2}^{R} \wedge \theta_{2}^{R}=\varphi_{1}^{R} \forall R=\{\circ, \Delta\}
$$

The geometry of the domain and the primary variables are approximated by nodal values and shape functions applying the isogeometric concept. For this purpose, the finite quadrilateral element presented in EISENTRäGER et al. [14] is used. The continuous field variables are thus described with the help of discrete nodal values and vector-valued shape functions, i.e. quadratic shape functions of Serendipity type $N^{i}$ with $i \in[1, N N]$ (see [40])

$$
\mathbf{a}(\boldsymbol{\xi})=\mathbf{N}(\boldsymbol{\xi}) \mathbf{a}^{e} \quad \mathbf{a}^{e}=\left[\begin{array}{lllllllllll}
u_{1}^{e 1} & u_{2}^{e 1} & u_{1}^{\Delta e 1} & u_{2}^{\Delta e 1} & w^{e 1} & \theta_{1}^{e 1} & \theta_{2}^{e 1} & \theta_{1}^{\Delta e 1} & \theta_{2}^{\Delta e 1} & \ldots & \theta_{2}^{\Delta e N}
\end{array}\right]^{\top}
$$

where $N N$ is the number of nodes per element, which in this case is eight, $\boldsymbol{\xi}$ are the natural coordinates in the space of shape functions, and $\mathbf{N}$ is the matrix of shape functions containing all $N^{i}$, described in [14]. The kinematical relations in Equation (26) are approcimated with the nodal degrees of freedom in the usual way.

$$
\left[\begin{array}{l}
\tilde{\varepsilon}_{\mathrm{MB}} \\
\tilde{\varepsilon}_{\mathrm{S}}
\end{array}\right]=\left[\begin{array}{l}
\mathbf{B}_{\mathrm{MB}} \\
\mathbf{B}_{\mathrm{S}}
\end{array}\right] \mathbf{a}^{e} \quad \mathbf{B}_{j}=\mathbf{D}_{j} \mathbf{N}
$$

where $\tilde{\boldsymbol{\varepsilon}}_{j} \forall j=\{\mathrm{MB}, \mathrm{S}\}$ denotes the approximated strain vectors in VoIGT notation [41], $\mathbf{B}_{j}$ are the differential operators for strains, where MB and S denote the coupled membrane-bending and shear state, and $\mathbf{D}_{j}$ is the differential operator of strains in natural coordinates which differs for $\mathrm{MB}$ or $\mathrm{S}$ state. The result of the finite element approximation is a linear system of equations on element level, containing the element stiffness matrix $\mathbf{K}^{e}$ and the residual force vector $\mathbf{f}^{e}$, including internal and external forces. Now, the individual constituents have now to be assembled over all element nodes and elements of the discretised structure.

$$
\mathbf{K}=\bigcup_{e=1}^{N E} \mathbf{K}^{e}=\bigcup_{e=1}^{N E}\left(\mathbf{K}_{\mathrm{MB}}^{e}+\mathbf{K}_{\mathrm{S}}^{e}\right) \quad \mathbf{a}=\bigcup_{e=1}^{N E} \mathbf{a}^{e} \quad \mathbf{f}=\bigcup_{e=1}^{N E} \mathbf{f}^{e}
$$

Applying the fundamental lemma of variational calculus results in a linear system of equations on the structural level.

$$
\mathbf{K a}=\mathbf{f} \quad \mathbf{K}=\mathbf{K}_{\mathrm{MB}}+\mathbf{K}_{\mathrm{S}} \quad \mathbf{f}=\int_{\Gamma_{\mathrm{N}}} \mathbf{N}^{\top} \mathbf{p} \mathrm{d} A_{\mathrm{N}}
$$

Herin, $\mathbf{K}$ is the stiffness matrix at structural level, given in [14]. The division of matrices is into coupled membranebending and shear state is introduced to implement a selective integration procedure to avoid artificial stiffening effects like shear locking. The integration procedure is here done by using the GAuss-LeGENDRE quadrature [42] where full integration is applied. As shown in [14], this is possible since the finite element presented is not sensitive to artificial stiffening (locking effects) at such high discretisation densities, where an aspect ratio of $A R=1$ is also used. Furthermore, $A_{\mathrm{N}}$ is the loaded area and $\mathbf{p}$ is load vector. In contrast to [14], the right-hand-side vector $\mathbf{f}$ can be simplified according to

Table 4: Characteristic parameters of the discretisation variation resulting through $h^{e}$-adaptation at the composite structure

\begin{tabular}{l|lllll}
\hline mesh & 1 & 2 & 3 & 4 & 5 \\
\hline$N E$ & 50 & 200 & 5,000 & 20,000 & 80,000 \\
$N G$ & 450 & 1,800 & 45,000 & 180,000 & 720,000 \\
$A R$ & 1 & 1 & 1 & 1 & 1 \\
\hline
\end{tabular}



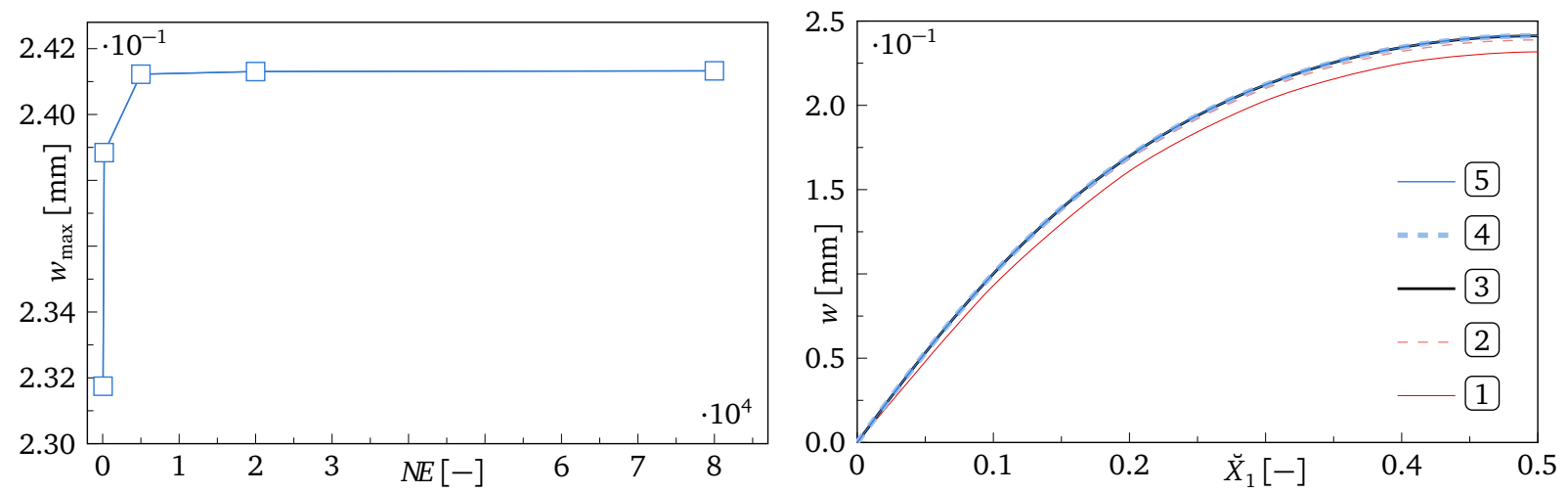

Figure 7: Convergence of maximum deflection in the center of the plate and the displacement along Path A-A during discretisation variation

the neglection of line loads, concentrated forces and moments as far as residual stresses. A detailed derivation of these equations based on the application of the principle of virtual work can be found in [14]. While pre- and postprocessing is done using FORTRAN, the processing is accomplished by using the ABAQUS solver as mentioned in [14]. To examine the accuracy of the FE solution, a convergence analysis by a $h^{e}$-adaptation is performed. Here, the edge lengths of the quadrilateral elements are varied, while the aspect ratio remains constant $A R=1 \forall \Omega_{\mathrm{XLWT}}^{e}$. The resulting number of elements and integration points are summarised in Table 4. The results are presented in Figure 7, where normalised length measures along the plate half along $\boldsymbol{e}_{1}$ are used to visualise the mesh-dependent deflection. Here, $\breve{X}_{1}=X_{1} / L_{1}$ and $\breve{X}_{2}=X_{2} / L_{2}$ holds true. $w_{\max }=w\left(X_{1} / 2, X_{2} / 2\right)$ is the central deflection, which is also the maximum deflection in the present case. It turns out that around 5,000 elements are sufficient for present calculations.

\subsection{Temperature dependent solutions}

During the operation lifetime in natural weathering, a photovoltaic module is subjected to temperatures ranging from approximately -20 to $+70^{\circ} \mathrm{C}$ [43], which are resulting from ambient temperature, wind speed and direction, radiation exchange and semiconductor efficiency loss to name the main influencing factors. Due to the strong temperature dependence of the encapsulant resulting in significant variation of the YounG's modulus of the core layer, temperature dependent studies are required. In the absence of availability of complete data of temperature dependent material parameters of EVA, we utilise the data gained from EITNER [29] for three different temperatures, $\vartheta=\left\{-40^{\circ} \mathrm{C},+23^{\circ} \mathrm{C}\right.$, $+80^{\circ} \mathrm{C}$ \}. Concerning the material parameters of glass and silicon, it is assumed that these are temperature independent in the present study. Since the PoIsson's ratio cannot take any value $\geq 0.5$ for isotropic materials in linear elasticity theory due to postulation of positive definiteness of the strain energy density, we used the value of 0.41 at EVA for temperatures deviating from $+23{ }^{\circ} \mathrm{C}$ in contrast to the work of EITNER [29]. Other details presented in Table 5 are the ratio of the shear moduli $\left(G R=G^{c} / G^{s}\right)$ confirming the statements made by NAUMENKo \& EREMEYEV [13] concerning the application range and the need of the XLWT for photovoltaic modules, and the normalised shear rigity parameter $\beta$ which gives an indication of the scope of application of composite structure theories and is determined as follows for symmetric three-layered composite structures.

$$
\beta=\sqrt{\frac{2\left[1-\left(v^{t}\right)^{2}\right] \kappa G^{c} L_{\min }^{2}}{E^{t} h^{t} h^{c}}\left[1+3\left(1+\frac{h^{c}}{h^{t}}\right)^{2}\right]} \quad L_{\min }=\min \left\{L_{1}, L_{2}\right\} \wedge \kappa=\kappa^{K}=1
$$

Comparing present structure to the structure analysed in EISENTRÄGER et al. [14] , the KIRCHHOFF theory can be applied at a temperature of $-40{ }^{\circ} \mathrm{C}$, and the Mindus theory delivers sufficiently accurate results at $+23^{\circ} \mathrm{C}$. The layerwise theory shows, in addition to the application at high temperatures $\left(+80^{\circ} \mathrm{C}\right)$, the widest range of application. It can be applied in all temperature ranges for any admissible variation of the shear rigidity parameter, what represents the main advantage of this theory,

For reasons of clarity, we present results of two paths along the composite structure for this temperature dependent analysis only. These paths, specified in Table 6, run along the two bisected in-plane directions of the composite structure. Results concerning kinematical measures are visualised in Figure 8. 
Table 5: Temperature dependent material parameters of encapsulant used for the core layer [29] and resulting ratios GR and $\beta$ of the composite structure

\begin{tabular}{l|lll}
\hline$\vartheta\left[{ }^{\circ} \mathrm{C}\right]$ & -40 & +23 & +80 \\
\hline$E\left[\mathrm{~N} / \mathrm{mm}^{2}\right]$ & 1019.04 & 7.90 & 0.52 \\
$v[-]$ & 0.41 & 0.41 & 0.41 \\
$G\left[\mathrm{~N} / \mathrm{mm}^{2}\right]$ & 361.36 & 2.80 & 0.18 \\
\hline$G R[-]$ & $1.29 \cdot 10^{-2}$ & $9.98 \cdot 10^{-5}$ & $6.57 \cdot 10^{-6}$ \\
$\beta[-]$ & 106.74 & 9.40 & 2.41 \\
\hline
\end{tabular}
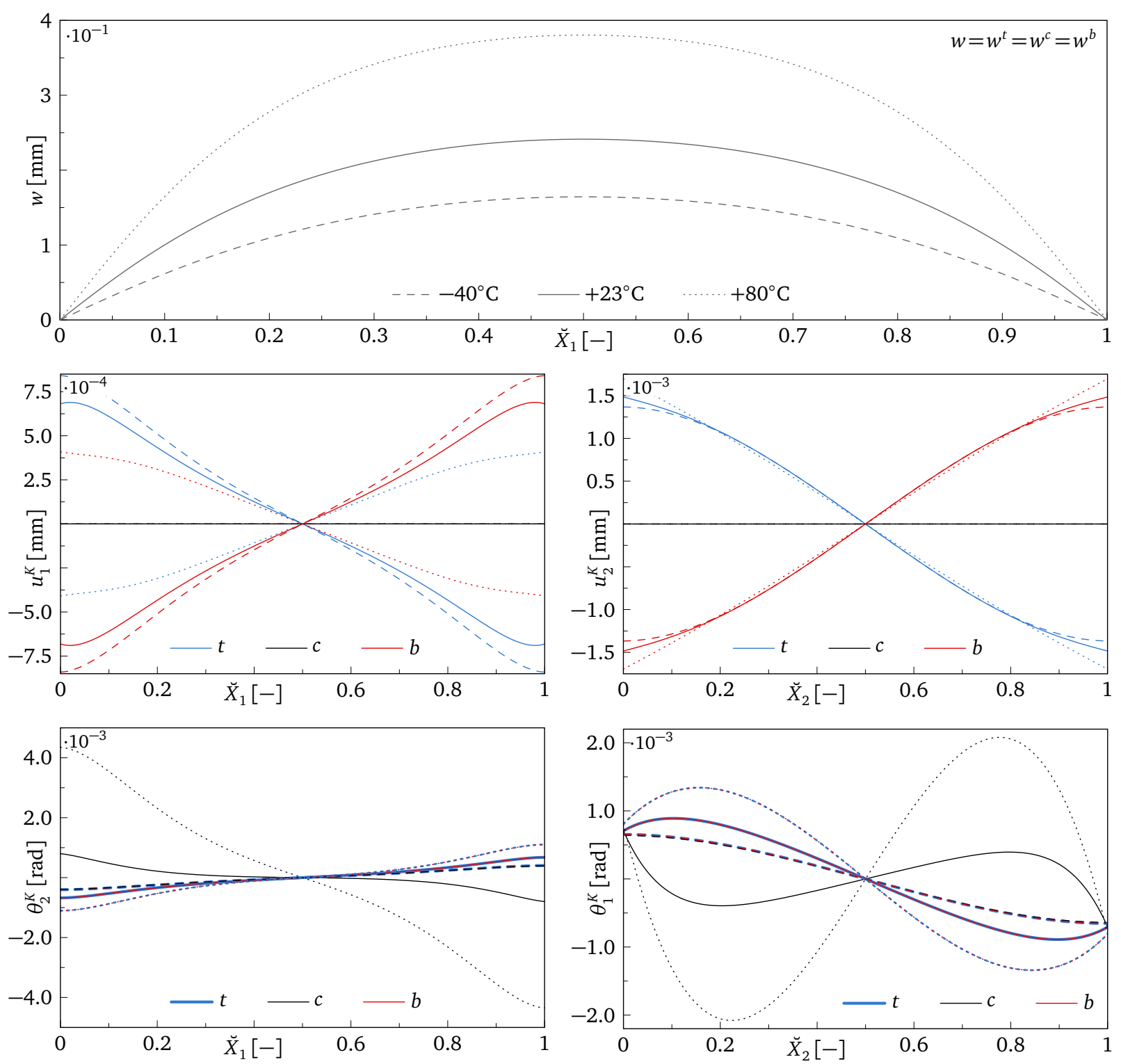

Figure 8: Deflections, in-plane displacements and cross-section rotations of top $(t)$, core $(c)$, and bottom layer $(b)$ along the Paths A-A $\left(\breve{X}_{1}\right)$ and B-B $\left(\breve{X}_{2}\right)$ at $-40^{\circ} \mathrm{C}$ (dashed line), $+23^{\circ} \mathrm{C}$ (solid line), and $+80^{\circ} \mathrm{C}$ (dotted line) 
Table 6: Path definitions for plots in Figuree 7 and 8 in compliance with origin of coordinate system from Figure 4

\begin{tabular}{l|ll}
\hline Path & Start $\left(X_{1}, X_{2}, X_{3}\right)$ & End $\left(X_{1}, X_{2}, X_{3}\right)$ \\
\hline A-A & $0, L_{2} / 2,0$ & $L_{1}, L_{2} / 2,0$ \\
B-B & $L_{1} / 2,0,0$ & $L_{1} / 2, L_{2}, 0$ \\
\hline
\end{tabular}

As to be expected, the deflection of the composite structure increases with rising temperature due to the decrease of stiffness of the core layer. Since the load applied is orthogonal and homogeneous, the maximum deflection can be found in the centre of the composite structure. Following values for the maximum deflection have been determined.

$$
w_{\max }\left(-40^{\circ} \mathrm{C}\right)=1.64 \cdot 10^{-1} \mathrm{~mm} \quad w_{\max }\left(+23^{\circ} \mathrm{C}\right)=2.41 \cdot 10^{-1} \mathrm{~mm} \quad w_{\max }\left(+80^{\circ} \mathrm{C}\right)=3.80 \cdot 10^{-1} \mathrm{~mm}
$$

Concerning the in-plane displacements, a boundary layer effect can be recognised which decay up to 5\% of the plate edge length. These displacements are more than two magnitudes of order smaller than the deflection. Due to the geometrical symmetry and the homogenoeus, orthogonal loading, there are no in-plane displacements of the core layer. The rotations in skin and core layers are equal at $-40{ }^{\circ} \mathrm{C}$. However, the rotation of the core layer runs opposite with respect to the rotations of the skin layers at higher temperatures. For more details and further discussion with respect to stress resultants, we refer to EISENTRÄGER et al. [14].

\section{Local stuctural analysis}

\subsection{Unit cell choice, geometry, and materials}

Considering a photovoltaic module, a planar periodicity can be identified. This mesoscopic periodicity is indicated in Figure 2, bottom left. The representative part is a unit cell indicated by every individual solar cell. The solar cell is surrounded by the encapsulant and the skin layers. This representative proportion $\mathfrak{B}_{i}^{\mathrm{UC}}$ of the photovoltaic module $\mathfrak{B}$ is considered, where following relations hold true.

$$
\mathfrak{B}_{i}^{\mathrm{UC}} \subset \mathfrak{B}
$$

$$
\mathfrak{B}_{i}^{\mathrm{UC}} \cap \mathfrak{B}_{j}^{\mathrm{UC}}=\emptyset
$$

sectional view $\left(X_{3}=0.5 L_{3}\right)$
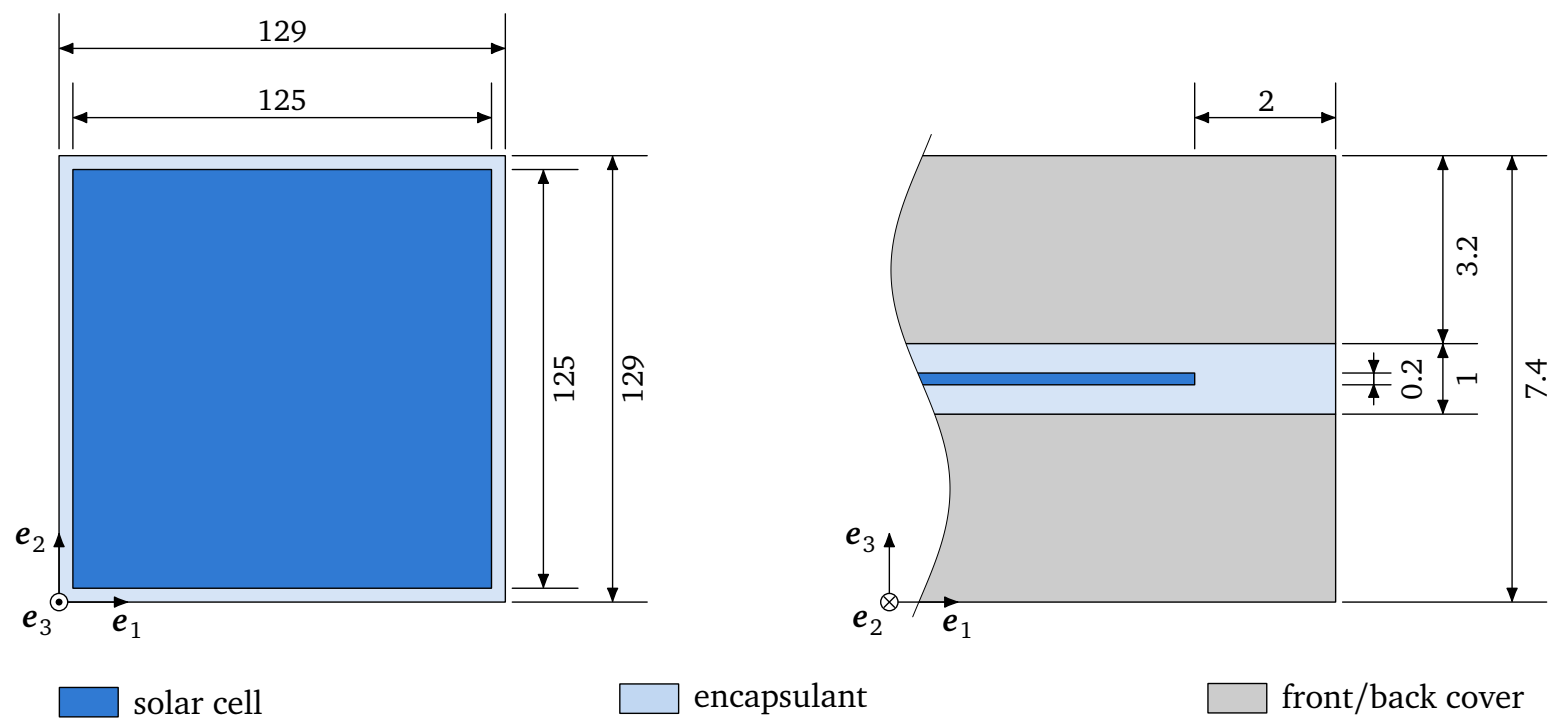

solar cell

encapsulant

front/back cove

Figure 9: Design and dimensions of the abstract unit cell $\tilde{\mathfrak{B}}_{i}^{\mathrm{UC}}$ used for local structural analysis (not to scale, dimensions in mm) 
For the sake of simplicity, this unit cell is also abtracted where we want to neglect all elelctrical contacts. Considering that the selected structure is only representative in the two plane directions, is dispensed with at this point on the term RVE and instead used the term unit cell [44]. The overall dimensions of the unit cell are $129 \times 129 \times 7.4 \mathrm{~mm}^{3}$ $\left(L_{1}^{\mathrm{UC}} \times L_{2}^{\mathrm{UC}} \times L_{3}^{\mathrm{UC}}\right)$ depicted in Figure 9 . The solar cell is centered vertically and horizontally. In plane, the solar cell is thus surrounded in each direction with $2 \mathrm{~mm}$ encapsulant material. In the direction of the skin layers, the distance between the solar cells and the skin layers is $0.4 \mathrm{~mm}$.

\subsection{Mechanical boundary value problem at local scale}

As only a time-invariant linear-elastic problem has been considered at global scale, it is sufficient to consider the quasi-static case at local scale, too. In contrast to the global problem, classical continuum theory can be applied at local scale. Basics on continuum mechanics can be found in many textbooks, for example [17, 19]. The starting point is the balance of linear momentum which leads us to the strong formulation of the local boundary value problem.

$$
\boldsymbol{\nabla} \cdot \boldsymbol{T}=\mathbf{0} \quad \text { with } \boldsymbol{T}=T_{i j} \boldsymbol{e}_{i} \otimes \boldsymbol{e}_{j} \quad \forall \boldsymbol{x} \in \tilde{\mathfrak{B}}_{i}^{\mathrm{UC}}
$$

Herein, $\boldsymbol{T}(\boldsymbol{x})$ is the CAUCHY stress tensor which is related to the infinitesimal strain tensor $\boldsymbol{E}$ by a constitutive law. Considering geometrical linearity, the strain tensor ist defined by the symmetric part of the displacement gradient.

$$
\boldsymbol{E}=\boldsymbol{\nabla}^{\mathrm{sym}} \boldsymbol{u} \quad \text { with } \boldsymbol{u}=u_{i} \boldsymbol{e}_{i} \quad \forall \boldsymbol{x} \in \tilde{\mathfrak{B}}_{i}^{\mathrm{UC}}
$$

Assuming linear elastic and isotropic material behaviour, the constitutive law can now be written as follows.

$$
\boldsymbol{T}=\mathcal{C}: \boldsymbol{w} \quad \text { with } \mathcal{C}=2 \mu \mathcal{I}^{\mathrm{sym}}+\lambda \mathbf{1} \otimes \mathbf{1}
$$

Herein, $\mathcal{C}$ refers to the fourth rank isotropic constitutive tensor in linear elasticity with Lamé's parameters $\lambda$ and $\mu$, which are related to the engineering parameters $E, v$, and $G$ in the isotropic case, see e.g. [17]. $\mathcal{I}^{\text {sym }}$ represents the symmetric fourth rank identity tensor, and $\mathbf{1}$ denotes the second rank identity. To this end, DiRICHLET

$$
\boldsymbol{u}=\boldsymbol{u}^{\star} \quad \forall \boldsymbol{x} \in \Gamma_{\tilde{u}}^{\mathrm{UC}}
$$

and NeumanN boundary conditions

$$
t^{\star}=\boldsymbol{T} \cdot \boldsymbol{n} \quad \forall x \in \Gamma_{T}^{\mathrm{UC}}
$$

are introduced where the following relations hold true.

$$
\Gamma^{\mathrm{UC}}=\Gamma_{\boldsymbol{T}}^{\mathrm{UC}} \cup \Gamma_{\boldsymbol{u}}^{\mathrm{UC}} \quad \Gamma_{\boldsymbol{T}}^{\mathrm{UC}} \cap \Gamma_{\boldsymbol{u}}^{\mathrm{UC}}=\emptyset \quad \Gamma^{\mathrm{UC}}=\partial \tilde{\mathfrak{B}}_{i}^{\mathrm{UC}}
$$

Here, $\boldsymbol{t}^{\star}$ is the prescribed stress vector and $\boldsymbol{u}^{\star}$ the displacement field. The superscript ${ }^{\star}$ denotes a prescribed, time-invariant measure. Both boundaries will be specified in chapter 5 .

\subsection{Spatial dicretization and convergence of unit cell}

For the discretisation of the unit cell, 20-noded hexahedron elements are used. Details concerning these standard brick elements of quadratic order with three translational degrees of freedom $\left(u_{i}\right)$ are given, for example, in [45]. Since this is a standard element, with regard to the numerical procedure we refer to basic textbooks like [40] or [46]. Again, a structured mesh was chosen to discretise the domain $\tilde{\mathfrak{B}}_{i}^{\mathrm{UC}}$. To check the suitability and convergence of the unit cell, three characteristic tests are used, as visualised in Figure 10. These tests are a tension, a shear, and a bending test. Here the prescribed $u_{i}$ needs to be small such that the geometrically linear theory remains valid. Regarding the convergence tests, a discretisation variation through $h^{e}$-adaption is performed using five different meshes where the mesh parameters are summarised in Table 7. On the convergence analysis, the average stresses are used for evaluation which are determined by using the the volume weighted average of respective (CAUCHY) stresses resulting at the GaUss points.

$$
\overline{\boldsymbol{T}}=\int_{\tilde{\mathfrak{B}}_{i}^{\mathrm{UC}}} \boldsymbol{T} \mathrm{d} V \quad \Rightarrow \quad \bar{T}_{i j}=\frac{1}{\sum_{G P=1}^{N G} V^{G P}} \sum_{G P=1}^{N G} T_{i j}^{G P} V^{G P} \quad \forall G P \in \tilde{\mathfrak{B}}_{i}^{\mathrm{UC}}
$$


Table 7: Characteristic values of the discretisation variation resultung through $h^{e}$-adaptation at the unit cell

\begin{tabular}{l|lllll}
\hline Mesh & 1 & 2 & 3 & 4 & 5 \\
\hline$N E$ & 245 & 2,420 & 29,575 & 149,769 & 299,538 \\
$N G$ & 6,615 & 65,340 & 798,525 & $4,043,763$ & $8,087,526$ \\
$A R_{\varnothing}$ & 49.93 & 13.92 & 3.54 & 1.82 & 6.11 \\
$A R_{\max }$ & 125.00 & 31.25 & 10.00 & 5.00 & 10.00 \\
\hline
\end{tabular}

Herein, the superscript $G P$ is running variable for the volume $V^{G P}$ associated to the corresponding Gauss point and $N G$ is the total number of Gauss points in the discretised domain of the unit cell $\tilde{\mathfrak{B}}_{i}^{\mathrm{UC}} . T_{i j}^{G P}$ is the stress at the individual Gauss point.

As can clearly be seen, around 300,000 finite elements are needed to achieve convergence of selected stress parameters. Nevertheless, the maximum number of elements used there is considered sufficiently adequate for the present investigations, since a further increase in the number of elements results in highly increased CPU times, which is not effective for the intended aim here to present an efficient solution strategy.

\section{Coupling of global and local structure}

\subsection{Projection strategy}

Towards a proper estimation of stresses in the unit cell, a coupling of global and local scales is required. In principle, approaches to scale coupling of plates or shells to 3D continua can be found in the literature, often referred to as FE ${ }^{2}$, see $[47,48,49,50]$. Common to these approaches is the use of FOSDT at global scale, which is not appropriate to solve
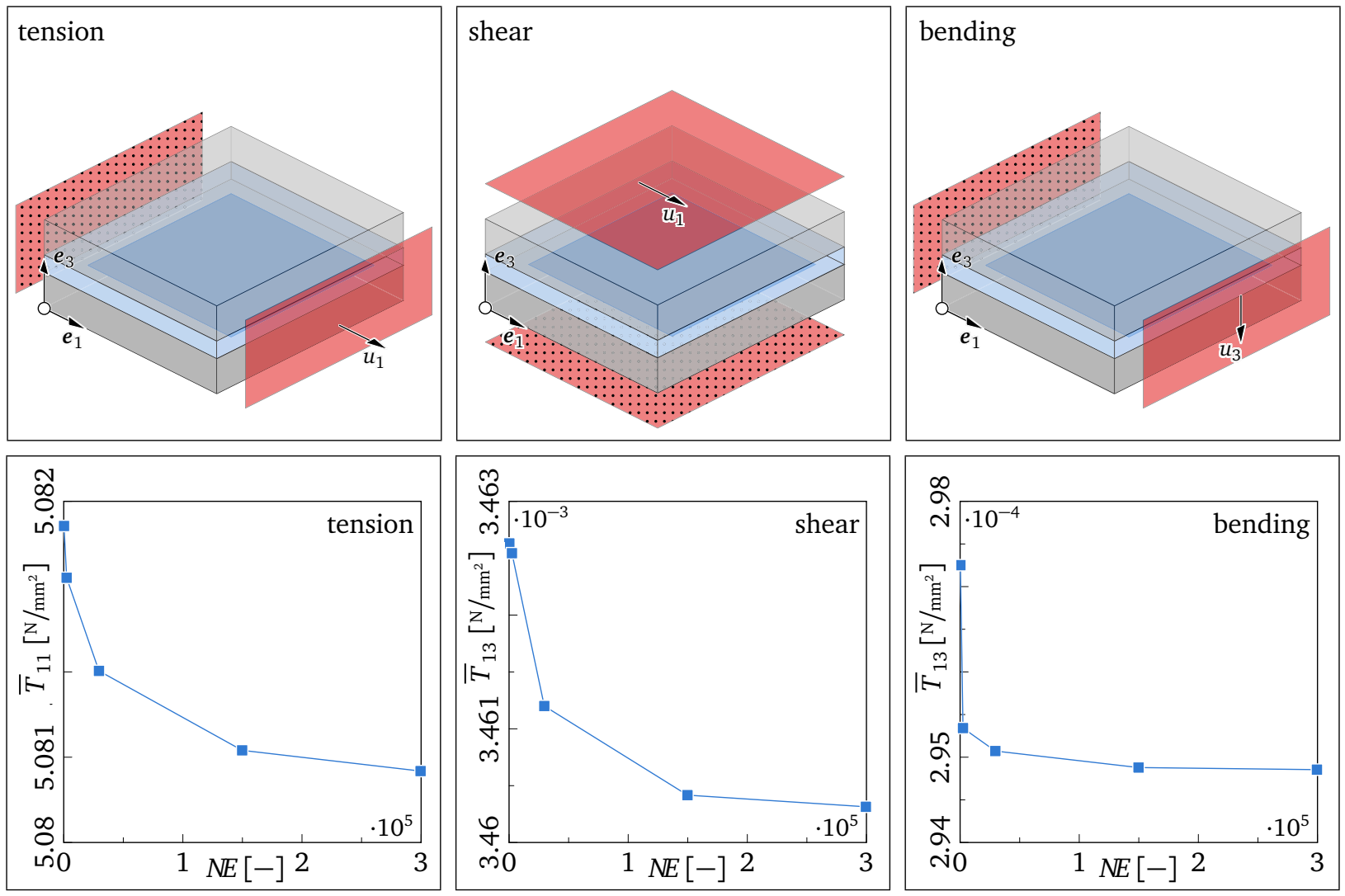

Figure 10: Convergence behaviour of the unit cell at three predefined tests for analyses at $23^{\circ} \mathrm{C}$ (pictures in upper row not to scale) 


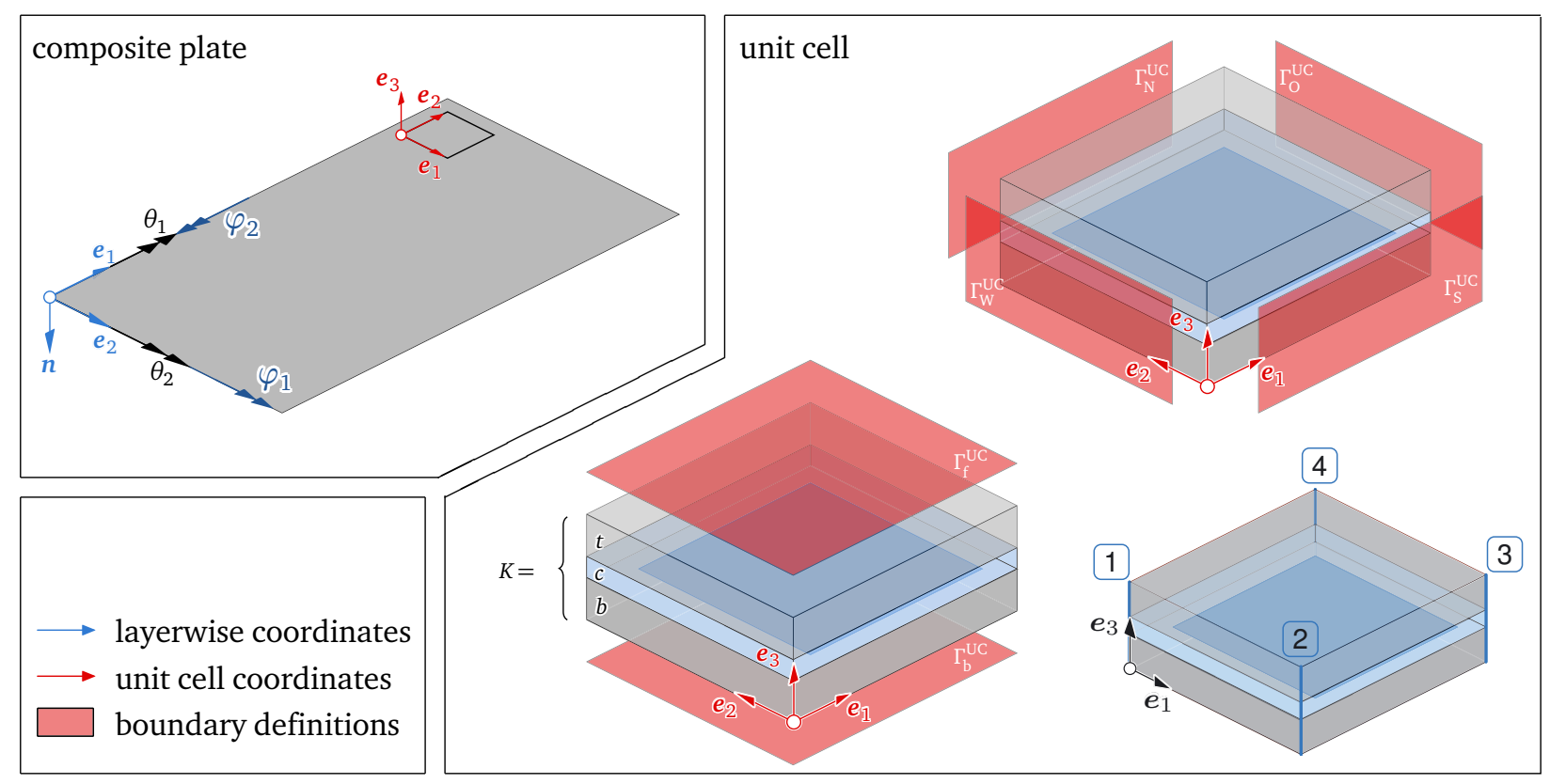

Figure 11: FE representation of the layerwise structure with coordinate systems (left) and unit cell with boundary definitions indicated (right)

structural problems for photovoltaic modules [9].However, these approaches have the objective to deliver effective material properties for the global scale. In the present study, our intention is just a unidirectional coupling from global to local scale for structural analysis. For the scale transition, the projection of the deformations is used here.

However, since there are no effective characteristics of a homogenization but stress results of realistic loading scenarios at local scale are of interest in this work, the deformations are projected directly from global to local scale such that both deformation gradients are equal. At global scale, a composite plate presented in Section 3 is considered, while at local scale a small volume like the one presented in Section 4 is investigated including the full thickness of the composite structure so that the scale separation is only verified in plane directions, but not in transverse direction. Due to absence of periodicity of the unit cell in $\boldsymbol{e}_{3}$ direction, the boundary conditions extracted from the global solution are projected to the side faces of the unit cell with normals in $\boldsymbol{e}_{1}$ and $\boldsymbol{e}_{2}$ directions only. Since the angle definitions of XLWT and the used commercial FE software differ, all rotational degrees of freedom are transferred to ABAQUS' definitions depicted as $\theta_{1}$ and $\theta_{2}$ in Figure 11 (see also [14] for this problem). For the sake of simplicity, in the context of the local FE modelling, a coordinate transformation is performed first. The orientation of the coordinate systems at global and local structures is illustrated in Figure 11 (left side). Due to the non-corresponding degrees of freedom of XLWT and the CAUCHY continuum [51], which is used for the unit cell, a conversion of the rotational degrees of freedom in translational degrees of freedom is performed. This can be determined through simple geometric relationships, glazing on the bottom and top edges of the respective layer which are based on the kinematic hypotheses of the underlying theory.

$$
u_{\alpha}\left(X_{1}, X_{2}, X_{3}\right)=u_{\alpha}\left(X_{1}, X_{2}\right)+X_{3} \theta_{\beta}\left(X_{1}, X_{2}\right) \quad u_{3}\left(X_{1}, X_{2}, X_{3}\right)=w\left(X_{1}, X_{2}\right) \quad \forall \alpha=\{1,2\} \wedge \alpha \neq \beta
$$

The resulting displacements are applied to all nodes of the elements lying at the side faces of the unit cell $\Gamma_{\mathrm{N}}^{\mathrm{UC}}, \Gamma_{\mathrm{S}}^{\mathrm{UC}}$, $\Gamma_{\mathrm{O}}^{\mathrm{UC}}, \Gamma_{\mathrm{W}}^{\mathrm{UC}}$ visualised in Figure 11, right hand side. This results in a pure displacement-driven formulation on local scale. For the sake of simplicity, the projection is done only for one unit cell position, where coordinates for both scales for present case study are given in Table 8 . For a clear connection of both scales, dimensions in different coordinate systems are color coded and visualised this way in Figure 11, also. Measures with a superscript index UC are related to the local scale. 


\subsection{Implementation and numerical utilization}

\subsubsection{DOF's of eXtended LayerWise Theory}

In contrast to the work of EISENTRÄGER et al. [14], a new vector of degrees of freedom is introduced, where the displacements and rotation angles of every layer are considered separately to get a clearer view of the projection. Considering the kinematical constraints given in [13] displayed in Equations (32) and (33), the rotations and in-plane displacements of the core layer can be represented by the degrees of freeom of the outer layers.

$$
\varphi^{c}=\frac{1}{h^{c}}\left[\boldsymbol{u}^{b}-\boldsymbol{u}^{t}-\frac{h^{b}}{2} \varphi^{b}-\frac{h^{t}}{2} \varphi^{t}\right] \quad \boldsymbol{u}^{c}=\frac{1}{2}\left[\boldsymbol{u}^{t}+\boldsymbol{u}^{b}+\frac{h^{t}}{2} \boldsymbol{\varphi}^{t}-\frac{h^{b}}{2} \boldsymbol{\varphi}^{b}\right]
$$

Taken into account that the angle definitions in XLWT theory (see [9, 13, 14]) and commercial FE programm systems do not correspond but are linked with $\varphi^{K}=\boldsymbol{\theta}^{K} \times \boldsymbol{n}$, the relations $\theta_{1}^{K}=-\varphi_{2}^{K}$ and $\theta_{2}^{K}=\varphi_{1}^{K}$ are introduced in Equation (42), where $\theta_{i}^{K}$ is the angle definition in FE codes visualised in Figure 11 (left side). The original vector of degrees of freedom from equation (42) can now be written in detailed manner. In summary, this vector now includes 13 degrees of freedom, which can be represented as follows.

$$
\mathbf{a}^{\mathrm{XLWT}}=\left[\begin{array}{c}
\mathbf{a}^{t} \\
\mathbf{a}^{c} \\
\mathbf{a}^{b} \\
w
\end{array}\right]
$$

$$
\mathbf{a}^{K}=\left[\begin{array}{llll}
u_{1}^{K} & u_{2}^{K} & \theta_{1}^{K} & \theta_{2}^{K}
\end{array}\right]^{\top} \quad \forall K=\{t, c, b\}
$$

The deflections are still assumed to be the same for every layer $\left(w=w^{K} \forall K=\{t, c, b\}\right)$ at any point $\boldsymbol{x}_{\ominus}=X_{\alpha} \boldsymbol{e}_{\alpha}$ of the composite structure like given in Equation (34). To avoid large amounts of data, all parameters of (57) are extracted at all $\boldsymbol{x}_{\ominus} \in g$ defined in Table 8 (global scale) for all $K=\{t, c, b\}$. That is legitimate since the plane dimensions $L_{\alpha}$ at local scale are much smaller than those at global scale.

\subsubsection{DOF's of Unit Cell}

The unit cell is based on the classical CAUCHY continuum where each material point is uniquely identifiable by $\boldsymbol{x}=X_{i} \boldsymbol{e}_{i}$. Each material point has three translational degrees of freedom $\boldsymbol{u}=u_{i} \boldsymbol{e}_{i}$. Independent rotations as they are taken into account in XLWT are not considered. The vector of dregrees of freedom is therefore given as follows.

$$
\mathbf{u}=\left[\begin{array}{lll}
u_{1} & u_{2} & u_{3}
\end{array}\right]^{\top}
$$

\subsubsection{Coordinate transformation}

Once having defined an appropriate representation of the vector of degrees of freedom within explicit parameters for all layers and extracted those parameters at defined corner edges, the transformation from XLWT composite structure to unit cell coordinate system has to be performed. Therefore the transformation matrix $\mathbf{T}_{\mathrm{cs}}$ is introduced, which maps the extracted vector $\mathbf{a}^{\mathrm{XLWT}}$ from Section 5.2.1 to the vector in unit cell coordinate system $\mathbf{a}_{\mathrm{P}}^{\mathrm{UC}}$ (Note color coding

Table 8: Definition of the four corner edges at the unit cell for scale couplingin red (position definition in XLWT coordinate system blue, see fig. 11)

\begin{tabular}{l|llll}
\hline corners $g$ & 1 & 2 & 3 & 4 \\
\hline$X_{1}$ & 0 & $L_{1}^{\mathrm{UC}}$ & $L_{1}^{\mathrm{UC}}$ & 0 \\
$X_{2}$ & 0 & 0 & $L_{2}^{\mathrm{UC}}$ & $L_{2}^{\mathrm{UC}}$ \\
$X_{1}$ & $1438 \mathrm{~mm}$ & $1438 \mathrm{~mm}$ & $1567 \mathrm{~mm}$ & $1567 \mathrm{~mm}$ \\
$X_{2}$ & $13 \mathrm{~mm}$ & $142 \mathrm{~mm}$ & $142 \mathrm{~mm}$ & $13 \mathrm{~mm}$ \\
\hline
\end{tabular}




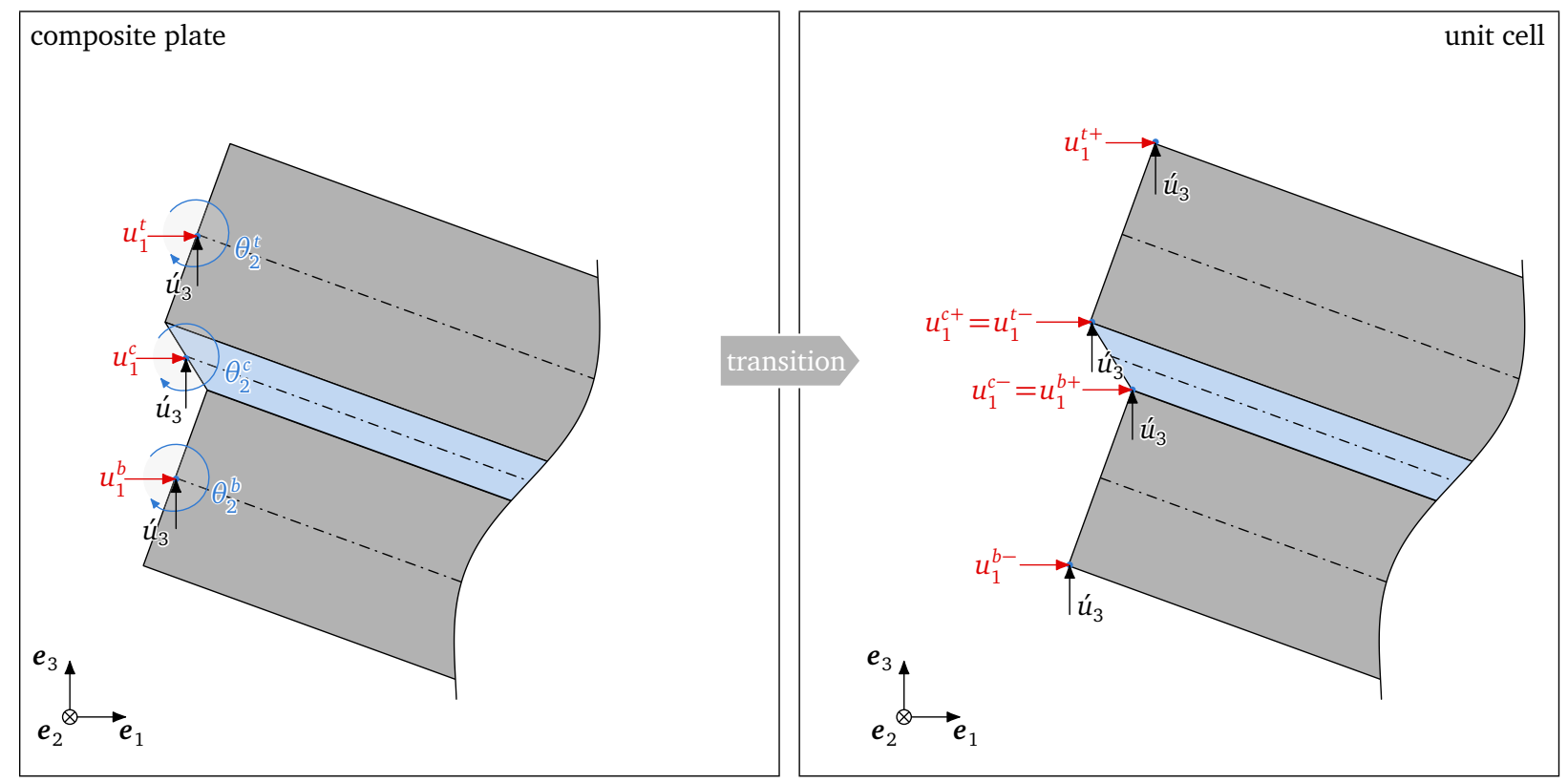

Figure 12: Exemplary visualization of parameter transition in $\boldsymbol{e}_{1}$-direction from composite structure to $3 \mathrm{D}$ at corner 1$]$ within the projection strategy

analogous to the coordinate systems colors depicted in Figure 11). This is done by the follwing equation.

$$
\begin{aligned}
& \mathbf{a}_{\mathrm{P}}^{\mathrm{UC}}=\mathbf{T}_{\mathrm{cs}} \mathbf{a}^{\mathrm{XLWT}} \\
& {\left[\begin{array}{l}
u_{1}^{t} \\
u_{2}^{t} \\
\theta_{1}^{t} \\
\theta_{2}^{t} \\
u_{1}^{c} \\
u_{2}^{c} \\
\theta_{1}^{c} \\
\theta_{2}^{c} \\
u_{1}^{b} \\
u_{2}^{b} \\
\theta_{1}^{b} \\
\theta_{2}^{b} \\
u_{3}^{b}
\end{array}\right]_{\mathrm{P}}\left[\begin{array}{ccccccc}
\mathbf{T} & \mathbf{0} & \mathbf{0} & \mathbf{0} & \mathbf{0} & \mathbf{0} & \mathbf{0} \\
\mathbf{0} & \mathbf{T} & \mathbf{0} & \mathbf{0} & \mathbf{0} & \mathbf{0} & \mathbf{0} \\
\mathbf{0} & \mathbf{0} & \mathbf{T} & \mathbf{0} & \mathbf{0} & \mathbf{0} & \mathbf{0} \\
\mathbf{0} & \mathbf{0} & \mathbf{0} & \mathbf{T} & \mathbf{0} & \mathbf{0} & \mathbf{0} \\
\mathbf{0} & \mathbf{0} & \mathbf{0} & \mathbf{0} & \mathbf{T} & \mathbf{0} & \mathbf{0} \\
\mathbf{0} & \mathbf{0} & \mathbf{0} & \mathbf{0} & \mathbf{0} & \mathbf{T} & \mathbf{0} \\
\mathbf{0} & \mathbf{O}^{\top} & \mathbf{O}^{\top} & \mathbf{0} & \mathbf{0} & \mathbf{0} & -1
\end{array}\right]\left[\begin{array}{c}
u_{1}^{t} \\
u_{2}^{t} \\
\theta_{1}^{t} \\
\theta_{2}^{t} \\
u_{1}^{c} \\
u_{2}^{c} \\
\theta_{1}^{c} \\
\theta_{2}^{c} \\
u_{1}^{b} \\
u_{2}^{b} \\
\theta_{1}^{b} \\
\theta_{2}^{b} \\
w
\end{array}\right] \quad \mathbf{0}=\left[\begin{array}{ll}
0 & 0 \\
0 & 0
\end{array}\right]}
\end{aligned}
$$

\subsubsection{Composite to $3 D$ transition}

The XLWT is based on a surface continuum including displacements and rotations. Since the CAUCHY continuum cannot handle independent rotations, a transformation of parameters from XLWT to 3D continuum is required. The approach used here is based on the needle hypothesis. It is assumed that a straight line normal to the midplane of a layer in unloaded state remains straight after applying a load, regardless if this line tilts or not during a loading at all admissible loading processes. For this, a layerwise extrapolation of the vector of degress of freedom starting from midplane of every layer is introduced. Resulting values are obtained at top (+) and bottom (-) faces of every layer. This is implemented by applying the projection matrix $\mathbf{P}_{23}$ to the vector of degrees of freedom $\mathbf{a}_{\mathrm{P}}^{\mathrm{UC}}$ resulting from previous section. The methodology is visualised exemplary in Figure 12. 


$$
\begin{aligned}
& \mathbf{u}_{\mathrm{P}}=\mathbf{P}_{23} \mathbf{a}_{\mathrm{P}}^{\mathrm{UC}} \\
& {\left[\begin{array}{l}
u_{1}^{t+} \\
u_{1}^{t-} \\
u_{2}^{t+} \\
u_{2}^{t-} \\
u_{1}^{c+} \\
u_{1}^{c-} \\
u_{2}^{c+} \\
u_{2}^{c-} \\
u_{1}^{b+} \\
u_{1}^{b-} \\
u_{2}^{b+} \\
u_{2}^{b-} \\
u_{3}^{b-}
\end{array}\right]_{\mathrm{P}}=\left[\begin{array}{cccc}
\mathbf{P}^{t} & \mathbf{0} & \mathbf{0} & \mathbf{0} \\
\mathbf{0} & \mathbf{P}^{c} & \mathbf{0} & \mathbf{0} \\
\mathbf{0} & \mathbf{0} & \mathbf{P}^{b} & \mathbf{0} \\
\mathbf{O}^{\top} & \mathbf{O}^{\top} & \mathbf{O}^{\top} & 1
\end{array}\right]\left[\begin{array}{c}
u_{1}^{t} \\
u_{2}^{t} \\
\theta_{1}^{t} \\
\theta_{2}^{t} \\
u_{1}^{c} \\
u_{2}^{c} \\
\theta_{1}^{c} \\
\theta_{2}^{c} \\
u_{1}^{b} \\
u_{2}^{b} \\
\theta_{1}^{b} \\
\theta_{2}^{b} \\
u_{3}
\end{array}\right]_{\mathrm{P}}} \\
& \mathbf{0}=\left[\begin{array}{llll}
0 & 0 & 0 & 0 \\
0 & 0 & 0 & 0 \\
0 & 0 & 0 & 0 \\
0 & 0 & 0 & 0
\end{array}\right] \\
& \mathbf{o}=\left[\begin{array}{llll}
0 & 0 & 0 & 0
\end{array}\right]^{\top} \\
& \mathbf{P}^{K}=\left[\begin{array}{cccc}
1 & 0 & 0 & +h^{K} / 2 \tan \\
1 & 0 & 0 & -h^{K} / 2 \tan \\
0 & 1 & +h^{K} / 2 \tan & 0 \\
0 & 1 & -h^{K} / 2 \tan & 0
\end{array}\right]
\end{aligned}
$$

Here $u^{K O}$ holds $\forall O=\{+,-\}$. Results are the boundary conditions in transverse direction at the edges of the unit cell for every front and back side of each layer, see Figure 11 bottom right. Due to the compatibility conditions of the XLWT, the following relationships hold true.

$$
u_{\alpha}^{t-} \stackrel{!}{=} u_{\alpha}^{c+} \quad u_{\alpha}^{c-} \stackrel{!}{=} u_{\alpha}^{b+}
$$

For reasons of numerical inaccuracy, an absolute tolerance measure which was fixed as a displacement deviation of $\Delta u_{\alpha}= \pm 10^{-9} \mathrm{~mm}$ is introduced here such that fullfilling the following four relations is sufficient for the transition.

$$
\begin{array}{ll}
\left|u_{i}^{t-}-u_{i}^{c+}\right| \stackrel{!}{\leq}\left|\Delta u_{\alpha}\right| & \left|u_{i}^{c-}-u_{i}^{b+}\right| \stackrel{!}{\leq}\left|\Delta u_{\alpha}\right| \\
\left|u_{i}^{c+}-u_{i}^{t-}\right| \stackrel{!}{\leq}\left|\Delta u_{\alpha}\right| & \left|u_{i}^{b+}-u_{i}^{c-}\right| \stackrel{!}{\leq}\left|\Delta u_{\alpha}\right|
\end{array}
$$

\subsubsection{Prevention of rigid body motions}

Since rigid body motions can generate stresses, it is necessary to prevent them. However, $\mathfrak{u}_{3}$ contains a portion of a rigid body motion. This proportion is to be eliminated here. For this purpose, the minimum deflection at the four points $g$ is determined. In consequence, the deflections at all four corners are upgraded with the deflection value predetermined. The procedure is specified by following expression, while it holds true for all positive load values at outer surface of the top layer of the global structure.

$$
\begin{aligned}
& u_{3}(g)=\dot{u}_{3}(g)+\dot{u}_{3}^{\min } \quad \dot{u}_{3}^{\min }=\min \left(\left|\dot{u}_{3}^{1}\right|,\left|\dot{u}_{3}^{2}\right|,\left|\dot{u}_{3}^{3}\right|,\left|\dot{u}_{3}^{4}\right|\right) \quad \forall g=\{1,2,2,3,4\} \\
& \mathbf{u}=\left[\begin{array}{lllllllllllll}
u_{1}^{t+} & u_{1}^{t-} & u_{2}^{t+} & u_{2}^{t-} & u_{1}^{c+} & u_{1}^{c-} & u_{2}^{c+} & u_{2}^{c-} & u_{1}^{b+} & u_{1}^{b-} & u_{2}^{b+} & u_{2}^{b-} & u_{3}
\end{array}\right]^{\top}
\end{aligned}
$$

At one corner edge, the deflection value is zero now.

\subsubsection{Layerwise bilinear interpolation}

Since the boundary conditions are now defined for the corner edge points of the unit cell for layer front and back sides only, the boundary conditions have to be determined in longitudinal and transverse directions to generate constraints over the boundaries $\Gamma_{\mathrm{N}}^{\mathrm{UC}}, \Gamma_{\mathrm{S}}^{\mathrm{UC}}, \Gamma_{\mathrm{O}}^{\mathrm{UC}}, \Gamma_{\mathrm{W}}^{\mathrm{UC}}$ defined in Section 5.1. For the sake of simplicity, a linear variation of previously determined parameters at selected point is assumed. The adoption of linear variation of the parameters in in-plane 
directions refers to thedifferent dimensions of the scales, here $L_{\alpha}^{\mathrm{UC}} \ll L_{\alpha}^{\mathrm{XLWT}}$, while the linear interpolation in transverse direction is legitimate due to the straight normal hypothesis (see MindLin [5]). To generate constraints described, a linear interpolation per layer is performed, where we differentiate between a interpolation for the in-plane displacements $u_{\alpha}$

$$
u_{\alpha}\left(X_{\alpha}, X_{3}\right)=\left[\begin{array}{r}
1-X_{\alpha} \\
X_{\alpha}
\end{array}\right]^{\top}\left[\begin{array}{ll}
u_{\alpha}^{K+}(0) & u_{\alpha}^{K+}\left(L_{\alpha}\right) \\
u_{\alpha}^{K-}(0) & u_{\alpha}^{K-}\left(L_{\alpha}\right)
\end{array}\right]\left[\begin{array}{r}
1-X_{3} \\
X_{3}
\end{array}\right]
$$

and for the deflections $u_{3}$

$$
u_{3}\left(X_{\alpha}, X_{3}\right)=\left[\begin{array}{r}
1-X_{\alpha} \\
X_{\alpha}
\end{array}\right]^{\top}\left[\begin{array}{ll}
u_{3}(0) & u_{3}\left(L_{\alpha}\right) \\
u_{3}(0) & u_{3}\left(L_{\alpha}\right)
\end{array}\right]\left[\begin{array}{r}
1-X_{3} \\
X_{3}
\end{array}\right]
$$

Composing the results of Equations (63) and (64) leads to a description of the displacement field $\mathbf{u}=\left[\begin{array}{lll}u_{1} & u_{2} & u_{3}\end{array}\right]^{\top} \forall \Gamma_{\mathrm{UC}}$ at every point of the side faces of the unit cell with normals in $\boldsymbol{e}_{1}$ and $\boldsymbol{e}_{2}$ direction for all layers. Compared to the original degrees of freedom of the global solution, the boundary conditions are completely identical at the edge points of the unit cell at every midplane of the unit cell only. Since the variation of the extracted variables at the edges of the unit cell along the plane directions is infinitesimal, this approach is legitimate. After all, the presented procedure leads to a pure displacement-driven formulation at local scale.

The whole procedure was implemented in the ABAQUS subroutine DISP using PYTHON scripts for calculation and automatisation and applied to the unit cell presented in Section 4.1 for the three different temperatures by using the affiliated temperature dependent material parameters for the encapsulation material according to Table 5. All following simulations are conducted using Mesh 5 presented in Table 7.

\section{Results and discussion}

\subsection{Preliminaries}

Here, the results of one sample position of the unit cell in the photovoltaic module are presented, where three characteristic temperatures concerning the encapsulant are considered. The unit cell position is given in Table 8 . Parameters of materials used are given Tables 1, and 5 respectively. Geometrical details are given in Figure 9. The load at the global structure remains small (see Section 3.3.2) since just the procedure is in the foreground. The evaluations are focussed on the UC-deformation and solar cell stresses where only basic conclutions of the solutions are drawn in the sequel.

\subsection{Unit cell deformation}

Analogous to the different displacements, rotations, and deflections of the composite structure due to varying temperatures, the unit cell also yields different implications. For this purpose, the deformation characteristics of the unit cell are juxtaposed comparatively. In Figure 13, the deformation state under identical mechanical loading for three different temperatures is visualised for the unit cell position defined in Table 8. It clearly shows that the deflection increases with increasing temperature. While the maximum deflection is $-1.58 \cdot 10^{-2} \mathrm{~mm}$ only at $-40^{\circ} \mathrm{C}$, it rises to $-5.48 \cdot 10^{-2} \mathrm{~mm}$ at $23^{\circ} \mathrm{C}$, and to $-8.15 \cdot 10^{-2} \mathrm{~mm}$ at $+80^{\circ} \mathrm{C}$ eventually. It turns out that the shear modulus of the core layer, which is decreasing with increasing temperature, has a strong influence on the deformation behaviour of the composite structure. While at very low temperatures almost identical rotations in all three layers are observed (compare with Section 3.5), the rotations of front and back layer are in the same direction and also of equal magnitude since we analyse a symmetric composite structure. The rotation of the core layer is contrarily. It can be seen clearly that a mutual sliding of the two skin layers occurs with increasing temperature. Therefore, it is required to use XLWT to analyse the deformation behaviour of the photovoltaic composite at global scale since the KIRCHHOFF theory and either the FOSDT cannot reproduce this structural behaviour at local scale after projection. 

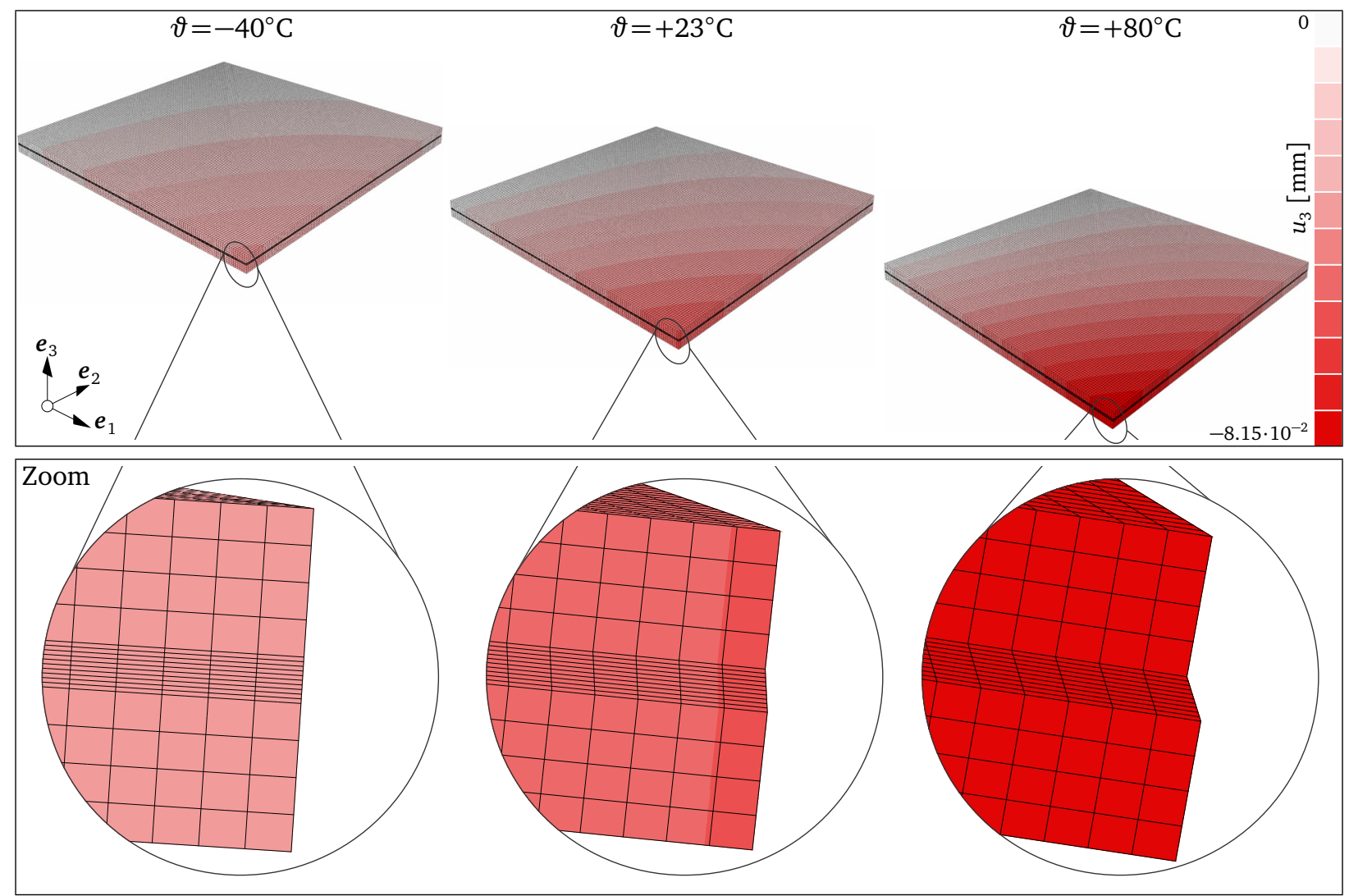

Figure 13: Deformation state of unit cell at studied temperatures with a focus on the displacements of the individual layers (magnified 250 times)

\subsection{Loading of solar cell}

The convential way for the assessment of brittle solids is applying a maximum stress or strain criteria. Exemplary strength values for the present material are given in [52], for example. We use the first and third principal stresses $T_{\mathrm{I}}$ and $T_{\mathrm{II}}$ prevailing in the embedded solar cell for evaluation comparatively. The principal stresses are determined by solving the symmetric eigenproblem [17] of the CAUcHY stress tensor, where the convention $T_{\mathrm{I}}>T_{\mathrm{II}}>T_{\mathrm{III}}$ holds true. The results of these stresses are visualised in Figure 14. Here, our analysis is reduced to surfaces with maximum and minimum stresses. The designation 'front side' indicates the surface of the solar cell at $X_{3}^{\mathrm{UC}}=3.8 \mathrm{~mm}$ and 'back side' at $X_{3}^{\mathrm{UC}}=3.6 \mathrm{~mm}$ in the context of the unit cell coordinate system. Maximum and minimum values of stresses can be found at $+80^{\circ} \mathrm{C}$. For $+23^{\circ} \mathrm{C}$ and $+80^{\circ} \mathrm{C}$ the distribution for $T_{\mathrm{I}}$ and $T_{\mathrm{III}}$ are qualitatively similar. The front sides are almost complete in tension state, and the back side is almost completely under pressure. Nevertheless, the tension and compression stresses at $+80^{\circ} \mathrm{C}$ are around $40 \%$ higher than at $+23^{\circ} \mathrm{C}$. This results from the larger deflection at higher temperatures (compare Figure 8). Comparing both results with the results at $-40^{\circ} \mathrm{C}$, one will find completely different stress distributions. Tension state can now be found at back side, while the front side yields compression. This occurs due to the strong difference in the elastic properties of the encapsulating material at temperatures below the glass transition regime. For example, the Young's modulus of EVA is increasing over several orders of magnitude of order. Now, the solar cells nearly follow the deformation which is prescribed by the skin layers. Resulting maximum or minimum stresses, respectively, range inbetween those values at $+23^{\circ} \mathrm{C}$ and $+80^{\circ} \mathrm{C}$.

In conclusion, these results emphasise the importance of temperature dependent analyses since the encapsulant material shows strongly varying mechanical behaviour, which results in completely different deformation and stress scenarios. 

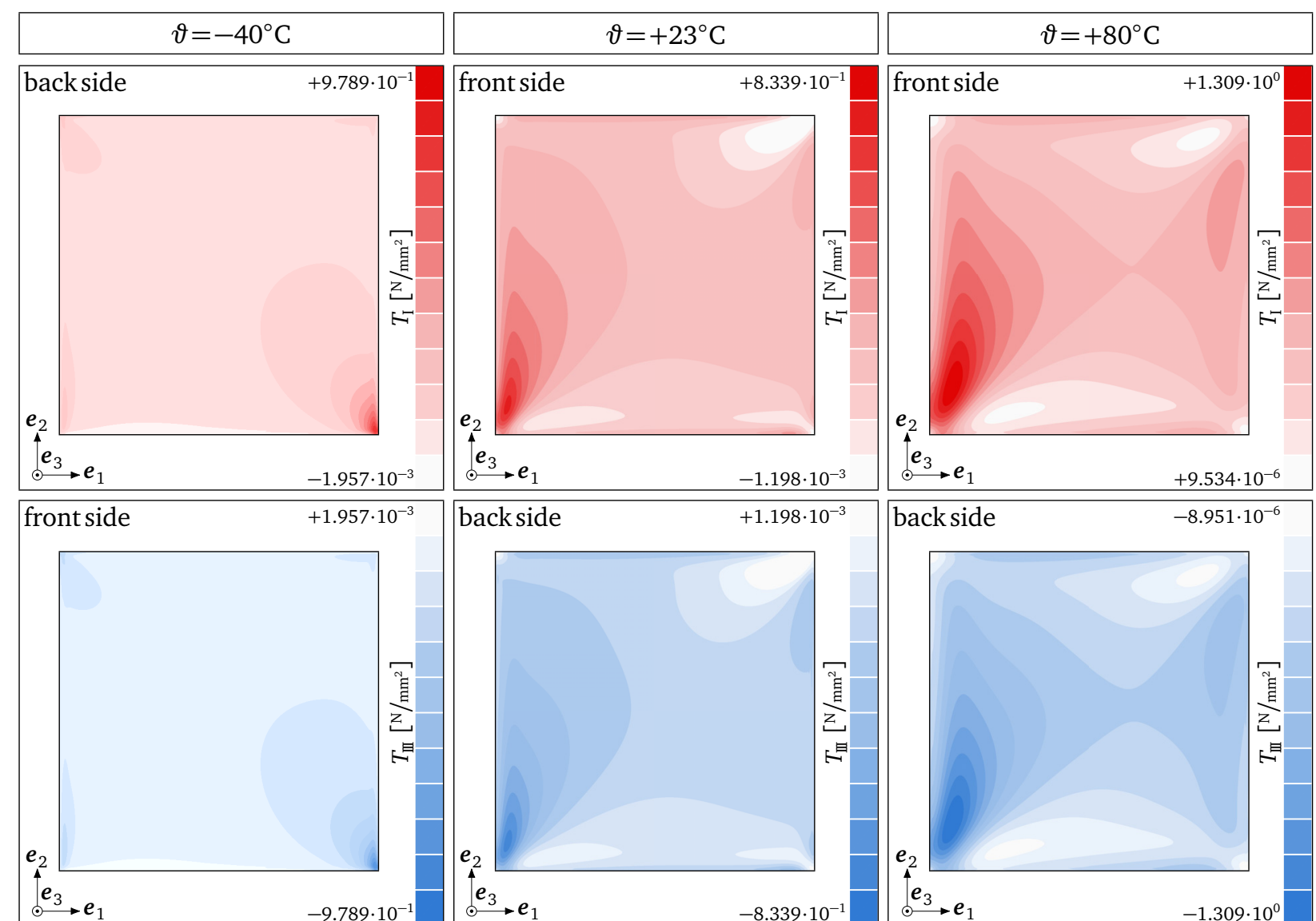

Figure 14: Visualization of principal stress distributions on front- and back side of the solar cell embedded in the unit cell at the studied temperatures

\section{Summary and outlook}

The aim of this study is to develop an efficient solution strategy to analyse the stress state of solar cells embedded in photovoltaic modules. The mechanical problem was therefore divided into two partial problems by a scale separation. Based on the eXtended LayerWise Theory developed by Naumenko \& Eremeyev [13] and the numerical implementation by EISENTRÄGER et al. [14], a solution of the structural mechanics problem at global scale was attained. At local scale a representative portion of a photovoltaic module with mesoscopic details is handled as three dimensional CAuchY continuum. Through periodic continuation of this portion in both plane directions a photovoltaic module results. The scale coupling is done by a projection. Here, a novel approach based on a transformation of degrees of freedom is developed, since independent rotational degrees of freedom cannot be handled at local scale. The whole procedure was applied succesfull at a case study.

XLWT provides an excellent opportunity for structural analysis at global scale of photovoltaic modules. The background is based on traceable assumptions. The fundamental assumption is based on the homogeneity postulate of the core layer. Here evidence is pending. One possibility for detection provides the homogenisation of the core layer where the geometrical features promise an orientation dependence of the mechanical behaviour. Comparing the range of application for present theory on global scale (valid if and only if $w_{\max } / H \leq 0.5$ ) with experimental results with more realistic load intensities (resulting in $w_{\max } / H \leq 10$ [53]), it is clear that a geometrically non-linear extension of the XLWT is required. At a minimum, large deflections should be considered to enlarge the scope.

The multiscale projection method for the analysis of embedded solar cells in a unit cell contains a simplifictaion where a bilinear interpolation is applied. From global solution, constraints in the sense of DirIchLET boundary conditions for 
the local structure analysis were extracted. For that, kinematical variables from specific coordinates for the position of a unit cell were used. Since the global theory gives also rotational degrees of freedom which cannot be applied in classical threedimensional continuum theory, a transformation of the kinematical variables was introduced where a linear variation of displacements in transverse direction is assumed. Since the interpolation in transverse direction is legitimate due to the underlying theory (Mindun kinematics and needle hypothesis), the interpolation in longitudinal directions requires further investigation for $L_{\alpha}^{\mathrm{UC}} \nless L_{\alpha}^{\mathrm{XLWT}}$, which is the case at large edge lengths of solar cells and /or small edge lengths of photovoltaic modules.

A local structural analysis was performed at a simplified unit cell, where metallic contacts were neglected. With knowledge of geometric details and material data, these structures can be easily taken under consideration. However, this in turn increases the computational effort. With regard to the numerical effort of present local analysis it can be shown that by linear extrapolation of the number of finite elements used for the unit cell, approximately $21.6 \cdot 10^{6}$ hexahedron elements with quadratic shape functions are required to analyse a a priori three dimensional model of a 72 solar cell photovoltaic module with comparable accuracy. The mechanical parameters of the homogeneous subsitute material model represented by an isotropic effective medium for the polycrystalline silicon solar cells are here based on a simple homogenization model captured by analytical approximations, the so called VoIGT-Reuss-HiLL average. For strength studies of polycrystalline silicon solar cells it is of prime importance to involve the material microstructure. This is of high relevance since polycrystal materials do not deform homogeneously but tend to reveal substantial inter- and transgranular stress-strain inhomogeneity since the individual grains are anisotropic and subject to different orientations. Further work should therefore focus on the implementation of a microstructure for the polycrystalline silicon solar cell. For this, knowledge of the crystal orientation distribution and crystal size distribution of the polycrystal is necessary. Only then it is possible to analyse cracks and fractures in solar cells almost realistic.

The methodology presented here provides an efficient and effective strategy to solve boundary value problems to capture the mechanical baheviour of photovoltaic modules through a sequential procedure of a deductive mulstiscale approach. This procedure is generally applicable to all admissible unit cells of a photovoltaic module while one have to loop this procedure sequentially or simultaneously over all $n$ unit cell positions of the underlying structure to gain results for the whole photovoltaic module. While in the present representation the procedure is introduced at a symmetric three-layered composite structure for the sake of brevity, it is also applicable to non-symmetric composite structures without loss of generality.

\section{Acknowledgement}

Financial support was provided by the German National Science Foundation (DFG) in the framework of the research training group 1554 'Micro-Macro-Interactions in Structured Media and Particle Systems'. This support is highly acknowledged. The authors would also like to thank Mykola Ievdokymov and Oleksandr Prygorniev for comments and advices to early versions of the subroutines created in FORTRAN and PYTHON. Special thanks goes to Johanna Eisenträger for reviewing the draft version of this contribution.

\section{Bibliography}

[1] M. Köntges, I. Kunze, S. Kajari-Schröder, X. Breitenmoser, B. Bjørneklett, The risk of power loss in crystalline silicon based photovoltaic modules due to micro-cracks, Solar Energy Materials and Solar Cells 95 (4) (2011) 1131-1137. doi:10.1016/j.solmat.2010.10.034.

[2] M. Sander, S. Dietrich, M. Pander, M. Ebert, J. Bagdahn, Systematic investigation of cracks in encapsulated solar cells after mechanical loading, Solar Energy Materials and Solar Cells 111 (2013) 82-89. doi:10.1016/j.solmat.2012.12.031.

[3] G. R. Kirchoff, Über das Gleichgewicht und die Bewegung einer elastischen Scheibe, Crelles Journal für die reine und angewandte Mathematik 40 (1850) 51-88. doi:10.1515/crll.1850.40.51.

[4] E. Reissner, The effect of transverse shear deformation on the bending of elastic plates, Journal of Applied Mechanics 12 (1945) 69-77.

[5] R. D. Mindlin, Influence of rotatory inertia and shear on flexural motions of isotropic, elastic plates, Journal of Applied Mechanics 18 (1951) 31-38.

[6] H. Altenbach, J. Altenbach, W. Kissing, Mechanics of Composite Structural Elements, Springer, Berlin · Heidelberg · New York, 2004. doi:10.1007/978-3-662-08589-9.

[7] J. N. Reddy, Mechanics of Laminated Composite Plates and Shells: Theory and Analysis, 2nd Edition, Taylor \& Francis, Boca Raton, 2004. 
[8] H. Altenbach, V. A. Eremeyev, K. Naumenko, On the use of the first order shear deformation plate theory for the analysis of three-layer plates with thin soft core layer, Zeitschrift für Angewandte Mathematik und Mechanik 95 (10) (2015) 1004-1011. doi:10.1002/zamm.201500069.

[9] J. Eisenträger, K. Naumenko, H. Altenbach, H. Köppe, Application of the first-order shear deformation theory to the analysis of laminated glasses and photovoltaic panels, International Journal of Mechanical Sciences 96-97 (2015) 163-171. doi:10.1016/j.ijmecsci.2015.03.012.

[10] F. Tornabene, N. Fantuzzi, M. Bacciocchi, E. Viola, Accurate inter-laminar recovery for plates and doubly-curved shells with variable radii of curvature using layer-wise theories, Composite Structures 124 (2015) 368-393. doi:10.1016/j.compstruct.2014.12.062.

[11] F. Tornabene, N. Fantuzzi, M. Bacciocchi, Higher-order structural theories for the static analysis of doubly-curved laminated composite panels reinforced by curvilinear fibers, Thin-Walled Structures 102 (1) (2016) 222-245. doi:10.1016/j.tws . 2016.01.029.

[12] C. Mittelstedt, W. Becker, Reddy's layerwise laminate plate theory for the computation of elastic fields in the vicinity of straight free laminate edges, Materials Science and Engineering A 498 (1) (2008) 76-80. doi:10.1016/j.msea.2007.10.122.

[13] K. Naumenko, V. A. Eremeyev, A layer-wise theory for laminated glass and photovoltaic panels, Composite Structures 112 (2014) $283-291$. doi:10.1016/j.ijmecsci.2015.03.012.

[14] J. Eisenträger, K. Naumenko, H. Altenbach, J. Meenen, A user-defined finite element for glass and photovoltaic panels based on a layer-wise theory, Composite Structures 133 (2015) 265-277. doi:10.1016/j.compstruct.2015.07.049.

[15] U. Eitner, S. Kajari-Schröder, M. Köntges, H. Altenbach, Thermal stress and strain of solar cells in photovoltaic modules, in: H. Altenbach, V. A. Eremeyev (Eds.), Shell-like Structures, Vol. 15 of Advanced Structured Materials, Springer, Berlin · Heidelberg, 2011 , pp. $453-468$. doi:10.1007/978-3-642-21855-2_29.

[16] M. Paggi, M. Corrado, M. Rodriguez, A multi-physics and multi-scale numerical approach to microcracking and power-loss in photovoltaic modules, Composite Structures 95 (2013) 630-638. doi:10.1016/j.compstruct.2012.08.014.

[17] H. Altenbach, Kontinuumsmechanik: Einführung in die materialunabhängigen und materialabhängigen Gleichungen, 2nd Edition, Springer Vieweg, Berlin · Heidelberg, 2012. doi:10.1007/978-3-642-24119-2.

[18] W. Lai, D. Rubin, E. Krempl, Introduction to Continuum Mechanics, 4th Edition, Butterworth-Heinemann, Oxford , 2010.

[19] A. Bertram, Elasticity and Plasticity of Large Deformations: An Introduction, 3rd Edition, Springer, Berlin · Heidelberg, 2012. doi : 10. $1007 / 978-3-642-24615-9$.

[20] A. Czanderna, F. Pern, Encapsulation of PV modules using ethylene vinyl acetate copolymer as a pottant: A critical review, Solar Energy Materials and Solar Cells 43 (2) (1996) 101-181. doi : 10.1016/0927-0248(95)00150-6.

[21] W. Voigt, Ueber die Beziehung zwischen den beiden Elasticitätsconstanten isotroper Körper, Wiedemanns Annalen 38 (1889) $573-587$. doi:10.1002/andp.18892741206.

[22] A. Reuss, Berechnung der Fließgrenze von Mischkristallen auf Grund der Plastizitätsbedingung für Einkristalle, Zeitschrift für Angewandte Mathematik und Mechanik 9 (1) (1929) 49-58. doi : 10.1002/zamm. 19290090104.

[23] R. Hill, Elastic properties of reinforced solids: some theoretical principles, Journal of the Mechanics and Physics of Solids 11 (5) (1963) 357-372. doi:10.1016/0022-5096(63)90036-X.

[24] J. den Toonder, J. van Dommelen, F. Baaijens, The relation between single crystal elasticity and the effective elastic behaviour of polycrystalline materials: theory, measurement and computation, Modelling and Simulation in Materials Science and Engineering 7 (6) (1999) 909-928. doi:10.1088/0965-0393/7/6/301.

[25] W. F. Hosford, The Mechanics of Crystals and Textured Polycrystals, Oxford University Press, New York, 1993.

[26] H.-J. Bunge, Über die elastischen Konstanten kubischer Materialien mit beliebiger Textur, Kristall und Technik 3 (3) (1968) $431-438$. doi:10.1002/crat.19680030309.

[27] H.-J. Bunge, Texture Analysis in Materials Science - Mathematical Methods, Butterworth \& Co., London · Boston · Sydney · Wellington · Durban · Toronto, 1982. doi:10.13140/RG.2.1.1721.1041.

[28] R. Cahn, P. Haasen, E. Kramer, Materials science \& technology. A comprehensive treatment. Vol. 9: Glasses and amorphous materials, Wiley-VCH, Weinheim, 1991.

[29] U. Eitner, Thermomechanics of photovoltaic modules, PhD thesis, Martin Luther University Halle-Wittenberg (2011). URL http://nbn-resolving.de/urn:nbn:de:gbv:3:4-5812

[30] P. A. Zhilin, Mechanics of deformable directed surfaces, International Journal of Solids and Structures 12 (9) (1976) 635-648. doi: 10.1016/0020-7683(76)90010-X.

[31] F. Cosserat, E. Cosserat, Théorie des corps déformables, A. Hermann et fils, Paris, 1909 URL http://jhir. library. jhu.edu/handle/1774.2/34209

[32] H. Altenbach, A directly formulated linear theory of viscoelastic plates and shells, Ingenieur-Archiv 58 (3) (1988) 215-228. doi : 10.1007/ BF00534332.

[33] H. Altenbach, P. A. Zhilin, The theory of simple elastic shells, in: R. Kienzler, I. Ott, H. Altenbach (Eds.), Theories of Plates and Shells: Critical Review and New Applications, Vol. 16 of Lecture Notes in Applied and Computational Mechanics, Springer, Berlin · Heidelberg, 2004, pp. 1-12. doi:10.1007/978-3-540-39905-6_1.

[34] S.-H. Schulze, M. Pander, K. Naumenko, H. Altenbach, Analysis of laminated glass beams for photovoltaic applications, International Journal of Solids and Structures 49 (1516) (2012) 2027-2036. doi:10.1016/j.ijsolstr.2012.03.028.

[35] M. Weps, K. Naumenko, H. Altenbach, Unsymmetric three-layer laminate with soft core for photovoltaic modules, Composite Structures 105 (2013) 332-339. doi:10.1016/j.compstruct.2013.05.029.

[36] H. Altenbach, An alternative determination of transverse shear stiffnesses for sandwich and laminated plates, International Journal of Solids and Structures 37 (25) (2000) 3503-3520. doi : 10 .1016/S0020-7683 (99) 00057-8.

[37] E. Winkler, Die Lehre von der Elasticität und Festigkeit mit besonderer Rücksicht auf ihre Anwendung in der Technik, Dominicus, Prag, 1867. URL http://digitalisate.bsb-muenchen.de/bsb10135085

[38] P. Pasternak, On a new method of analysis of an elastic foundation by means of two foundation constants (in russian), Gosudarstvennoe Izdatelstvo Literaturi po Stroitelstvu i Architekture, Dominicus, Moskva, 1954.

[39] Standard IEC 61215: Crystalline silicon terrestrial photovoltaic (PV) modules - Design qualification and type approval, International Electrotechnical Commission, Genf (2nd edition (2005)). 
[40] O. J. Zienkiewicz, R. L. Taylor, The Finite Element Method for Solid and Structural Mechanics, 6th Edition, Elsevier Butterworth-Heinemann, Oxford, 2005.

[41] W. Voigt, Lehrbuch der Kristallphysik (mit Ausschluss der Kristalloptik), Springer, Wiesbaden, 1966, Reproduktion des 1928 erschienenen Nachdrucks der ersten Auflage von 1910. doi:10.1007/978-3-663-15884-4

[42] H. R. Schwarz, N. Köckler, Numerische Mathematik, 8th Edition, B.G. Teubner, Wiesbaden, 2011. doi:10.1007/978-3-8348-8166-3.

[43] M. Koehl, M. Heck, S. Wiesmeier, J. Wirth, Modeling of the nominal operating cell temperature based on outdoor weathering, Solar Energy Materials and Solar Cells 95 (7) (2011) 1638-1646. doi:10.1016/j.solmat.2011.01.020.

[44] H.-J. Böhm, A short introduction to continuum micromechanics, in: H.-J. Böhm (Ed.), Mechanics of Microstructured Materials, Vol. 464 of International Centre for Mechanical Sciences (CISM), Springer, Vienna, 2004, pp. 1-40. doi : 10.1007/978-3-7091-2776-6_1.

[45] G. Dhondt, The Finite Element Method for Three-Dimensional Thermomechanical Applications, wiley, Chichester, 2004.

[46] M. A. Crisfield, Non-Linear Finite Element Analysis of Solids and Structures. Volume 1: Essentials, John Wiley \& Sons, Chichester, 1991.

[47] M. Geers, V. Kouznetsova, W. Brekelmans, Multi-scale computational homogenization: Trends and challenges, Journal of Computational and Applied Mathematics 234 (7) (2010) 2175-2182. doi:10.1016/j . cam.2009.08.077.

[48] S. Klinkel, F. Gruttmann, W. Wagner, A continuum based three-dimensional shell element for laminated structures, Computers \& Structures 71 (1) (1999) 43-62. doi : 10.1016/S0045-7949(98)00222-3.

[49] C. Helfen, S. Diebels, A numerical homogenisation method for sandwich plates based on a plate theory with thickness change, Journal of Applied Mathematics and Mechanics (ZAMM) 93 (2-3) (2013) 113-125. doi:10.1002/zamm. 201100173.

[50] C. Helfen, S. Diebels, Numerical multiscale modelling of sandwich plates, Technische Mechanik 32 (2-5) (2012) 251-264.

[51] A.-L. Cauchy, Note sur les séries convergentes dont les divers termes sont des fonctions continues d'une variable réelle ou imaginaire, entre des limites données, in: Euvres complètes, Vol. 12 of Series 1, Gauthier-Villars, Paris, 14 mars 1853, pp. 30-36, (published 1900). doi:10.1017/CB09780511702433.004.

[52] S. Schoenfelder, A. Bohne, J. Bagdahn, Mechanical strength of mono- and multicrystalline silicon wafers, in: Proceedings of the $18^{\text {th }}$ Workshop on Crystalline Silicon Solar Cells and Modules: Materials and Processes, National Renewable Energy Laboratory, Vail, Colorado, 2008, pp. $187-190$.

[53] M. Aßmus, S. Jack, K.-A. Weiß, M. Köhl, Measurement and simulation of vibrations of PV-modules induced by dynamic mechanical loads, Progress in Photovoltaics: Research and Applications 19 (6) (2011) 688-694. doi:10.1002/pip. 1087. 